\title{
Optimal monetary policy with heterogeneous money holdings
}

\author{
Francesco Lippi ${ }^{\mathrm{a}, \mathrm{b}, *}$, Stefania Ragni ${ }^{\mathrm{b}}$, Nicholas Trachter $^{\mathrm{c}}$ \\ ${ }^{\text {a }}$ EIEF, Italy \\ ${ }^{\mathrm{b}}$ University of Sassari, Italy \\ ${ }^{\mathrm{c}}$ Federal Reserve Bank of Richmond, United States
}

Received 28 January 2014; final version received 22 May 2015; accepted 4 July 2015

Available online 10 July 2015

\begin{abstract}
We study the optimal anticipated policy in a pure-currency economy with flexible prices and a nondegenerate distribution of money holdings. The economy features a business cycle and lump-sum monetary injections have distributional effects that depend on the state of the cycle. We parsimoniously characterize the dynamics of the economy and study the optimal regulation of the money supply as a function of the state under commitment. The optimal policy prescribes monetary expansions in recessions, when insurance is most needed by the cash-poor unproductive agents. Conversely, the optimal policy prescribes monetary contractions during booms, so that the inflationary effect of the occasional expansions is undone.
\end{abstract}

(c) 2015 Elsevier Inc. All rights reserved.

JEL classification: E50

Keywords: Heterogeneous agents; Redistributive monetary policy; Pure currency; Precautionary savings; Friedman rule

\footnotetext{
We thank Fernando Alvarez, Philip Barrett, Aleks Berentsen, Francisco Buera, Ricardo de O. Cavalcanti, Isabel Correia, Piero Gottardi, Christian Hellwig, Hugo Hopenhayn, Patrick Kehoe, Ricardo Lagos, David Levine, Fabrizio Mattesini, Guido Menzio, John Moore, Ezra Oberfield, Nicola Pavoni, Facundo Piguillem, B. Ravikumar, Tom Sargent, Rob Shimer, Alp Simsek, Balasz Szentes, Aleh Tsyvinski, Harald Uhlig, Neil Wallace, Randall Wright, and Bill Zame for useful discussions. We are grateful to several seminar participants. The views expressed in this article are those of the authors and do not necessarily represent the views of the Federal Reserve Bank of Richmond or the Federal Reserve System. Previous version of the paper was circulated under the title "State dependent monetary policy". Part of the research for this paper was sponsored by the ERC advanced grant 324008.

* Corresponding author.

E-mail address: f.lippi@uniss.it (F. Lippi).
} 


\section{Introduction}

We study the optimal monetary policy in a competitive flexible-price economy where infinitylived agents are subject to idiosyncratic productivity shocks and money is valued in equilibrium due to anonymity. The state of this economy is described by the wealth distribution, i.e. the distribution of money holdings, which evolves through time following the history of shocks and determines the value of money and aggregate output. Our objective is to characterize how to optimally regulate the money supply as a function of the state of the economy, what we call an optimal state-dependent monetary policy. A key feature of the setup is that monetary policy affects the wealth distribution, as in Wallace (1984) or Berentsen et al. (2005) and many other monetary models whose first principles are explicitly spelled out. Although the propagation of such redistributive effects of monetary policy is often "muted" by means of appropriate assumptions for the sake of tractability, as in Lucas (1990), Shi (1997), or Lagos and Wright (2005), in this paper we use an analytically tractable setup that allows us to study the role of systematic monetary policy taking fully into account the dynamics of the wealth distribution. The key assumption for tractability, following Scheinkman and Weiss (1986), is that we consider the simplest economy with time varying wealth distribution, namely one with two types of agents. We see this as a convenient starting point to study the interactions between the dynamics of the wealth distribution and monetary policy.

We think the question is interesting because it is novel in the theory and because the analysis provides a framework to interpret the large monetary expansions, sometimes observed during deep recessions, with a mechanism that is completely different from the canonical one relying on sticky prices. ${ }^{1}$ The properties of an optimal monetary policy in models where incomplete markets and heterogeneous agents allow for a potential redistributive role of monetary policy were first studied by Levine (1991). They have since been explored in a variety of contexts by Kehoe et al. (1992), Imrohoroglu (1992), Shi (1999), Bhattacharya et al. (2005), Molico (2006), Manuelli and Sargent (2010), Algan et al. (2011), and Rocheteau et al. (2015), for example. A common feature of these models is a tension between the benefits of a contractionary policy, i.e. one that yields an efficient return on money as under Friedman's rule, and the benefits of an expansionary policy, which provides partial insurance to cash-poor agents. A novelty of this paper is that, while previous models focused on a constant rule, i.e., seeking the optimal constant rate of monetary expansions, we consider a state-dependent monetary policy in which the rate of monetary expansion depends on the state of the economy.

Our model extends Scheinkman and Weiss's (1986) analysis, which assumes a constant money supply, by letting the government control the money supply through lump-sum transfers. ${ }^{2} \mathrm{We}$ provide a characterization of the price of money and of aggregate production in terms of the policy rule in a competitive equilibrium. We adopt an ex-ante welfare criterion and characterize an efficient monetary policy by solving a Ramsey problem. Our results cast some light on the interactions between the dynamics of the wealth distribution and the optimal anticipated policy. The main policy choice is a tradeoff between providing insurance (through monetary expansions) and ensuring an efficient return on savings (through monetary contractions). As argued

\footnotetext{
1 The assumption of flexible prices is useful to emphasize the workings of the redistributive role of monetary policy, and to distinguish it from the better understood mechanism that arises with sticky prices.

2 As in Levine (1991) we assume that the government does not know which agent is productive, so that the transfers are equal across agents. See Kehoe et al. (1992) for a thorough discussion of this assumption and in particular Levine (1991) for a derivation of the equal-treatment restriction from first principles.
} 
by Wallace (2014) this is a central tradeoff of several "general monetary economies", i.e. environments where money is essential, there are non-insurable shocks and money holdings are non-degenerate. ${ }^{3}$ In particular, in our setup the importance of the insurance motive varies with the state of the economy, so that a state-dependent monetary policy allows for a significant improvement compared with a constant policy. An expansionary monetary policy is efficient in recessions, when the poor and unproductive economic agents benefit from some redistribution of wealth. Surprisingly, in spite of the occasional large monetary expansions in states where the insurance motive is large, the optimal policy neutralizes the inflationary effect of these expansions by contracting the money supply in states where the insurance motive is small. In this way the state-dependent nature of the optimal policy allows for the provision of insurance when mostly needed without severely distorting the expected return of the asset. Thus, although our setup creates a potentially beneficial role for monetary expansions, the optimal rule prescribes an almost complete undoing of the inflationary effects of those expansions, and it implements a policy that brings the expected return on money as close as possible to the constant rate prescribed by the Friedman rule.

Our analysis is related to Molico (2006), who considers a search model of money with a non-degenerate distribution of cash holdings, showing that mild monetary expansions can be beneficial. In his model randomly matched agents may exchange goods for money. The price paid by the buyer results from bargaining and depends on the amount of money held by each agent upon entering the pairwise meeting. Therefore, the distribution of money is non-degenerate and monetary injections, via lump-sum transfers, can improve the terms of trade of poor buyers. Related results in the context of search models of money and mechanism design are obtained by Berentsen et al. (2005), Green and Zhou (2005), Deviatov and Wallace (2012), and Wallace (2013). The most important departure from Molico's analysis is that we restrict attention to an economy with only two types of agents, so that the wealth distribution (i.e. the state space) remains tractable. Because of this assumption we can study a policy that depends on the distribution of money holdings, while Molico focuses on a constant policy. A key feature of our model is that business cycles, and the magnitude of fluctuations, depend on the tightness of the borrowing constraint. More precisely, unlike other models in which it is the borrowing constraint itself which varies over the business cycle, e.g. because of the evolving value of the collateral as in Kiyotaki and Moore (1997), in our model it is the shadow value of the borrowing constraint which evolves with the business cycle. Even though our borrowing constraint is constant, its shadow value indicates that it is tighter in downturns (i.e. the value of money is higher in recessions). As a result inflation (the inverse of the return on money) is positively correlated with aggregate activity, thus generating a "Phillips curve". This result relates to Guerrieri and Lorenzoni (2009) and Guerrieri and Lorenzoni (2011) who explore the effects of borrowing constraints on business cycles in a model with liquid assets. Similar to their papers, as the borrowing constraint becomes tighter, economic fluctuations become more severe in our model. The relative simplicity of our setup allows us to investigate the optimal provision of liquidity. Our analysis is also related to Algan et al. (2011), who characterize the output-inflation tradeoff in a flexible price economy with incomplete markets and persistent wealth inequality among agents. While the setups are similar, the focus is different as monetary policy is treated as an exogenous parameter in these models.

\footnotetext{
3 To the best of our knowledge there are few models that provide a characterization of optimal policy under these assumptions. Rocheteau et al. (2015) is a recent interesting case.
} 
The model also illustrates a novel mechanism to interpret high frequency changes in the velocity of money (the ratio between GDP and real balances). This mechanism explains why money growth is not equal to inflation at every point in time, in spite of the fact that the two growth rates are equal in the long run. This suggests that the dynamics of the wealth distribution may help to understand the behavior of velocity, a result that is reminiscent of the segmented asset market literature as in e.g., Alvarez et al. (2009).

The paper is organized as follows. Section 2 presents the model. Section 3 shows how to characterize the monetary equilibrium. Section 4 analyzes the value of money in equilibrium under a state-dependent rule and compares it with the one with a constant money rule. Section 5 defines an ex ante welfare criterion and studies the best regulation of the money supply. Section 6 concludes.

\section{The model}

This section describes the model economy: agents' preferences, production possibilities, and markets. Two benchmark economies are discussed: the (efficient) allocation under complete markets and the optimal monetary policy with no uncertainty. We also establish that value functions and allocations are homogeneous in the exogenous parameters of interest; this is useful as it allows us to reduce the dimensionality of the problem by an appropriate normalization.

We consider two types of infinitely lived agents (with a large mass of agents of each type), indexed by $i=1,2$, and assume that at each point in time only one type of agent can produce. We further restrict attention to the case where agents of the same type play the same action at every point in time so that we can discuss the model in terms of two representative agents, one of each type. Because there are two agents in this economy, we can solve the model by looking at the problem from the perspective of agent one (i.e., $i=1$ ). Let $\mathcal{I}_{t}=\{0,1\}$ denote the productive type of the agent at time $t$. When $\mathcal{I}_{t}=1$ the agent can produce and transforms labor into consumption one for one; we label this agent as productive. When $\mathcal{I}_{t}=0$ the agent cannot produce; in this case we label the agent as unproductive. The productivity of labor is state dependent: the duration of productivity spells is random, exponentially distributed, with mean duration $1 / \lambda>0$. Money is distributed at each time $t$ between the two agents so that $m_{t}^{1}+m_{t}^{2}=M_{t}$. The growth rate of the money supply at time $t$ is $\mu_{t}$; then, the money supply follows $M_{t}=M_{0} e^{\int_{0}^{t} \mu_{j} d j}$, with $M_{0}$ given. As in Scheinkman and Weiss (1986) and Levine (1991), we let the individual state of an agent be private information, precluding agents from issuing private debt. ${ }^{4} \mathrm{~A}$ key assumption is that agents face a borrowing constraint restricting their unique savings instrument, money, to be non-negative. Because of the assumption of anonymity, fiscal policy has limited powers in this setup. ${ }^{5}$

Let $\rho>0$ denote the time discount rate. Each agent chooses consumption $c_{t}$, labor supply $\ell_{t}$, and depletion of money balances $\dot{m}_{t}^{1}$ in order to maximize her expected discounted utility,

\footnotetext{
4 Having a large mass of agents of each type is important for the argument as it implies that a single agent cannot infer the productive state of a different agent given his own state.

5 As common to many monetary models the distinction between fiscal and monetary policy is somewhat arbitrary, as first showed by Wallace (1981): if taxation is held constant, monetary policy affects the real present value of government liabilities. In the online Appendix we discuss what allocations can be achieved using tax policy under various assumptions about government powers (commitment vs. no commitment), types of available taxes (lump-sum vs. distortionary), and government knowledge about the state (agent's type observable vs. not observable).
} 


$$
\max _{\left\{c_{t}, \ell_{t}, \dot{m}_{t}^{1}\right\}_{t=0}^{\infty}} \mathbb{E}_{0}\left\{\int_{0}^{\infty} e^{-\rho t}\left(\ln c_{t}-\ell_{t}\right) d t\right\}
$$

subject to the constraints

$$
\begin{array}{rr}
\dot{m}_{t}^{1} \leq\left(\ell_{t}+\tau_{t}-c_{t}\right) / \tilde{q}_{t} & \text { if } \mathcal{I}_{t}=1 \\
\dot{m}_{t}^{1} \leq\left(\tau_{t}-c_{t}\right) / \tilde{q}_{t} \text { and } \ell_{t}=0 & \text { if } \mathcal{I}_{t}=0 \\
m_{t}^{1} \geq 0, \quad \ell_{t} \geq 0, \quad c_{t} \geq 0, \quad m_{0}^{1}, \mathcal{I}_{0} \text { given }, &
\end{array}
$$

where $\tilde{q}_{t}$ denotes the price of money, i.e., the inverse of the consumption price level, $\tau_{t}$ denotes a government lump-sum transfer to each agent, and where expectations are taken with respect to the productivity process defining $\mathcal{I}_{t}$ and $M_{t}$ conditional on time $t=0$.

A monetary policy with $\mu_{t}>0$ is called expansionary, a policy with $\mu_{t}<0$ is called contractionary. It is immediate that when the money supply is constant for all $t$ (i.e., $\mu_{t}=0 \forall t$ ) the economy is the one analyzed by Scheinkman and Weiss (1986). The monetary policy $\mu_{t}$ determines the transfers to the agents $\tau_{t}$ through the government budget constraint,

$$
\tilde{q}_{t} \mu_{t} M_{t}=2 \tau_{t} .
$$

The government transfer scheme implies that in the case of a contractionary policy, agents must use their money holdings to pay taxes (i.e. $\tau_{t}<0$ ). The "tax solvency" constraint, $m_{t}^{1} \geq 0$, imposes this restriction. Notice that in the continuous time characterization of the model the tax solvency constraint coincides with the borrowing constraint.

Note that the government cannot differentiate transfers across agent types. This follows from the assumption that the identity of the productive type is not known to the government. Levine (1991) shows in a similar setup that, because of anonymity, the best mechanism is linear and resembles monetary policy. Moreover, notice that the restriction of lump-sum transfers is such that we do not allow the planner to use the transfer scheme to motivate agents to truthfully reveal the level of their money stock. ${ }^{6}$ As widely done in the literature, we make this assumption to keep things as simple as possible.

Next we state two important remarks. The first one characterizes a symmetric efficient allocation with complete markets (the proof is standard so we omit it):

Remark 1. Assume complete markets and an ex-ante equal probability of each productive state. The symmetric efficient allocation prescribes the same constant level of consumption, $c_{t}=1$ for all $t$.

Thus, without borrowing constraints, the efficient allocation solves a static problem, and it encodes full insurance: Agents consume a constant amount (since ex-ante agents are equal) and aggregate output is constant.

The second remark characterizes the optimal monetary policy in the case of no uncertainty. This helps highlighting the essential role of uncertainty in our problem. In particular, consider the case where each agent oscillates deterministically between productive and unproductive cycles

\footnotetext{
6 This possibility is explored in Andolfatto (2010, 2013) and Wallace (2013). There, the transfer is composed of two terms: A lump-sum term and one that is increasing in the money stock of the agent. Because of this term, it seems possible to construct a transfer scheme where agents truthfully report their money stock.
} 
of length $T$. Without loss of generality, for the characterization of the stationary equilibria, let us assume that the economy starts in period $t=0$ with the agent being productive and holding no money, so that $m_{0}^{1}=0$. We have:

Remark 2. Consider a deterministic production cycle of length $T$. The symmetric efficient allocation, $c_{t}=1$ for all $t$ is attained by deflating at the rate of time preference $\mu_{t}=-\rho$ for all $t$.

A proof is available in Appendix B.1. This remark, together with the efficient allocation described in Remark 1, shows that without uncertainty this economy replicates the result of the optimality of the "Friedman rule" in Townsend (1980) and Bewley (1980).

To conclude notice that, by inspection of the agent problem presented in equations (1) to (4) and the evolution of money supply (i.e., $M_{t}=M_{0} e^{\int_{0}^{t} \mu_{j} d j}$ ), it is seen that the problem is homogeneous on $\left\{\lambda, \rho, \mu_{t}\right\}$ : allocations (the flows) are homogeneous of degree 0 while prices and values (the stocks) are homogeneous of degree minus 1 . This follows since the Poisson rate of changing states $\lambda$, the discount rate $\rho$, and the monetary expansion rate $\mu_{t}$ are all being measured with respect to a time unit (e.g., per year). Therefore, after normalizing by $\lambda$, the model has only two parameters: the normalized discount rate, $\rho / \lambda$, and the normalized money growth rate, $\mu_{t} / \lambda$. As a result, once we treat $\mu_{t}$ as a policy instrument, the model has only one exogenous parameter: the normalized discount rate $\rho / \lambda$.

\section{Characterization of monetary equilibrium}

This section defines and characterizes a monetary equilibrium. We show that the equilibrium amounts to a system of differential equations (and boundary conditions), and that the history of shocks is summarized by the wealth distribution. Finally we show that permanent monetary contractions cannot be sustained in equilibrium.

We look for an equilibrium where the price of money depends on the whole history of shocks, as encoded in the current values of the money supply, the distribution of money holdings, and the current state of productivity; that is, we let $\tilde{q}_{t}=\tilde{q}\left(M_{t}, m_{t}^{1}, \mathcal{I}_{t}\right)$. With a slight abuse of notation this implies $c_{t}=c\left(M_{t}, m_{t}^{1}, \mathcal{I}_{t}\right), \ell_{t}=\ell\left(M_{t}, m_{t}^{1}, \mathcal{I}_{t}\right)$, and $\dot{m}_{t}^{1}=\dot{m}^{1}\left(M_{t}, m_{t}^{1}, \mathcal{I}_{t}\right)$. As usual the nominal variables are homogenous of degree one in the level of money. With this in mind, we simplify the state space by letting $\hat{q}\left(z_{t}, \mathcal{I}_{t}\right)=M_{t} \tilde{q}\left(M_{t}, m_{t}^{1}, \mathcal{I}_{t}\right)$ denote the price of money when the agent holds $z_{t} \equiv \frac{m_{t}^{1}}{M_{t}}$ share of total money balances-a measure of the wealth distributionwhere $z_{t} \in[0,1]$. Likewise, the consumption and labor supply rules are homogeneous of degree zero in the level of the money supply, $c^{p}\left(z_{t}\right)=c\left(M_{t}, m_{t}^{1}, 1\right), c^{u}\left(z_{t}\right)=c\left(M_{t}, m_{t}^{1}, 0\right)$, and $\ell^{p}\left(z_{t}\right)=\ell\left(M_{t}, m_{t}^{1}, 1\right)$.

Let $x_{t}$ denote the wealth share in the hands of the unproductive agent. Note that this variable will record discrete jumps every time the identity of the productive type changes: whenever the identity of the productive type switches, the state $x$ jumps. That is, if $x=z$ before the state switches, then $x=1-z$ after it the switch occurs. We allow the planner to choose a Markovian monetary policy $\mu_{t}$ that is a continuous function of the state $x \in[0,1]$. As a result, $x_{t}$ summarizes the whole history of the economy and, without loss of generality, $\mu_{t}=\mu\left(x_{t}\right)$. Given the symmetry of the problem we let $q\left(x_{t}\right)$ denote the price of money in terms of consumption units, which occurs when the unproductive type assets are $x_{t}$. That is, if agent 1 holds wealth $z_{t}$, $q\left(x_{t}\right)=\hat{q}\left(z_{t}, 0\right)$ if she is unproductive and $q\left(x_{t}\right)=\hat{q}\left(1-z_{t}, 1\right)$ when she is productive. Next we define a monetary equilibrium. 
Definition 1. Given the continuous policy rule $\mu\left(x_{t}\right)$, the initial level of money supply $M_{0}$, the initial productivity status $\mathcal{I}_{0}$, the initial distribution of money holdings $x_{0}$, a monetary equilibrium is a price function $\tilde{q}_{t}=\frac{1}{M_{t}} q\left(x_{t}\right)$, with $q:[0,1] \rightarrow \mathbb{R}^{+}$and a stochastic process $x_{t}$ with values in $[0,1]$ such that, for all $t$, consumers maximize expected discounted utility (equation (1)) subject to (2), (3) and (4), and the market clearing constraint $c^{p}\left(1-x_{t}\right)+c^{u}\left(x_{t}\right)=\ell\left(1-x_{t}\right)$ and the government budget constraint are satisfied.

From now on we omit the time index $t$ to simplify the notation. A straightforward result is that permanent monetary contractions cannot be implemented in equilibrium. We state this result in the next lemma.

Lemma 1. There is no monetary equilibrium where $\mu(x)<0$ for all $x$. Moreover, all monetary equilibria must satisfy $\mu(0) \geq 0$.

See Appendix B.2 for a proof. The economics of this result is simple. As the length of the unproductive spell cannot be bounded above, there is a nonzero probability that a poor unproductive agent fails to cover her tax obligations. The only way she can fulfill her tax obligations is by keeping half of the money stock and not trading for goods. Because of no trade, money has no value (i.e. $q(x)=0$ for all $x$ ), there is no monetary equilibrium, and the allocation is autarkic. Moreover for any rule, including those that may allow for monetary contractions, the money growth rate cannot be negative at $x=0$. This is immediate since when $x=0$ unproductive agents hold no assets and are unable to cover their tax obligations.

Solving the model requires characterizing the marginal value of money given by the Lagrange multipliers for $\dot{m}^{1}$ in the problem defined in (1). Let $\tilde{p}\left(M, m^{1}\right)$ and $\tilde{u}\left(M, m^{1}\right)$ denote the un-discounted multipliers associated with the constraints in equations (2) and (3), respectively, so that, e.g., $\tilde{u}\left(M, m^{1}\right)$ measures the marginal value of money for agent 1 when the money supply is $M$, her wealth share is $m^{1} / M$, and she is unproductive. Likewise, $\tilde{p}\left(M, m^{1}\right)$ measures the marginal value for agent 1 when her wealth share is $m^{1} / M$ and she is productive. Using the homogeneity in the level of money $M$, we can write $\tilde{u}\left(M, m^{1}\right)=u(z) / M$ and $\tilde{p}\left(M, m^{1}\right)=p(z) / M$.

Combining the first order conditions with respect to $\ell$ and $c^{u}$ gives

$$
p(z)=q(x), \quad \frac{1}{c^{p}(z)}=\frac{p(z)}{q(x)}, \quad \text { and } \quad \frac{1}{c^{u}(z)}=\frac{u(z)}{q(x)},
$$

where $z$ is the share of money in the hands of the agent and $x$ is the share of money in the hands of the unproductive agent. These conditions equate marginal costs and benefits of an additional unit of money. The first two equations apply when the agent is productive (i.e. $\mathcal{I}=1$ ). The first one states that the marginal benefit of an additional unit of money, $p(z)$, equals the cost of obtaining that unit, i.e. the disutility of work to produce and sell a consumption amount $q(x)$. The second equation states that the marginal cost of the foregone unit of money, which is $p(z)$, equals the marginal benefit, which is given by the product of the price $q(x)$ (consumption per unit of money) times the marginal utility of consumption $1 / c^{p}(z)$. Notice that combining these two equations implies that $c^{p}(z)=1$ for all $z$. Finally, the third equation applies when the agent is unproductive (i.e., $\mathcal{I}=0$ ) and states that the marginal cost of the forgone unit of money $u(z)$, equals the benefit that is given by the additional units of consumption that can be bought with it: the product of the price $q(x)$ times the marginal utility of consumption $1 / c^{u}(z)$. 
The following functions define the evolution of money holdings,

$$
\dot{z}^{u}(z)=h^{u}(z) \quad \text { and } \quad \dot{z}^{p}(z)=h^{p}(z)
$$

where $h^{u}(z)$ is the change in the share of money holdings of an unproductive agent holding a share $z$, and $h^{p}(z)$ is the analogue for a productive agent. It is immediate that $h^{u}(z)+h^{p}(1-z)=0$. Consider the law of motion for $z$ by type 1 when unproductive,

$$
h^{u}(z)=\mu(x)\left(\frac{1}{2}-z\right)-\frac{c^{u}(z)}{q(x)}=\mu(x)\left(\frac{1}{2}-z\right)-\frac{1}{u(z)},
$$

where we used the budget constraint of the unproductive agent, equation (3), the government budget constraint, and the first order condition in equation (6).

For any $z \in(0,1)$ the marginal value of money for productive and unproductive agents, $p(z)$, and $u(z)$, solve a system of differential equations, which is the continuous time counterpart of the discrete time Euler equations,

$$
\begin{aligned}
& (\rho+\mu(x)) p(z)=p^{\prime}(z) h^{p}(z)+\lambda(u(z)-p(z)), \\
& (\rho+\mu(x)) u(z)=u^{\prime}(z) h^{u}(z)+\lambda(p(z)-u(z)) .
\end{aligned}
$$

The derivation is standard so we omit it. To provide some intuition consider the first equation: When the agent is productive and holds a share of money $z$, the value flow, discounted by the nominal rate $(\rho+\mu(x))$, is equal to the change in the marginal value due to the evolution of her money holdings, $p^{\prime}(z) h^{p}(z)$, and to the expectations of the change in value in case the state switches and the agent becomes unproductive: $\lambda(u(z)-p(z))$.

To complete the description of the equilibrium we provide the boundary condition for the marginal values of money $p(z)$ and $u(z)$. The boundary occurs when the unproductive agent has no money. In this case an unproductive agent spends the whole money transfer to finance her consumption, so that $h^{u}(0)=h^{p}(1)=0 .{ }^{7}$ The budget constraint gives that the consumption of an unproductive agent with no money is $\lim _{z \rightarrow 0} c^{u}(z)=\tau(0)=q(0) \mu(0) / 2$. Using equation (6),

$$
\lim _{z \rightarrow 0} u(z)=\frac{2}{\mu(0)},
$$

with $\lim _{z \rightarrow 0} u(z)=+\infty$ if $\lim _{x \rightarrow 0} \mu(x)=0$, and where the limit obtains because of Inada conditions. This is an important result in our analysis. An expansionary policy provides an upper bound to the marginal utility of money because the agent enjoys a positive consumption even with no wealth. If there is no money growth when the unproductive agent is poor (i.e., when $\mu(x) \rightarrow 0$ as $x \rightarrow 0$ ), the agent is not able to consume in poverty and therefore Inada conditions imply that her marginal utility diverges. Also, evaluating equation (9) at $z=1$ and $x=0$ gives

$$
u(1)=\left(1+\frac{\rho}{\lambda}+\frac{\mu(0)}{\lambda}\right) p(1) .
$$

Notice that $\mu(0)$, the money growth rate when the unproductive agent has zero wealth, appears in both boundaries. An implication is that the choice of the money growth rate simultaneously affects the insurance needs of the unproductive agents and the production incentives of the productive agents.

\footnotetext{
7 We provide a formal proof of this statement in Section 4.
} 


\section{The value of money in equilibrium}

The previous analysis showed that allocations in a monetary equilibrium are fully characterized by the Lagrange multipliers, $u(z)$ and $p(z)$, that solve the system of Euler equations and associated boundary conditions. This section characterizes the properties of these multipliers, measuring the "value of money" to the productive and unproductive agents, under two rules for the money supply. The first rule is state independent, i.e., equal to a constant non-negative money growth value $\mu \in[0,+\infty)$. The second rule assumes that the money growth rate is a continuous function of the wealth share of the unproductive agent: $\mu(x)$. We show that allowing the rule to respond to $x$ has a substantive impact on the shape of the value of money. This feature will play a crucial role in the optimal regulation of the money supply studied in Section 5.

Using equations (9)-(10) we can define the following system:

$$
\begin{aligned}
& p^{\prime}(z)=\frac{(\rho+\lambda+\mu(1-z)) p(z)-\lambda u(z)}{h^{p}(z)}, \\
& u^{\prime}(z)=\frac{(\rho+\lambda+\mu(z)) u(z)-\lambda p(z)}{h^{u}(z)} .
\end{aligned}
$$

The first equation describes the marginal value of money for a productive agent holding $z$, so that $x=1-z$, i.e., monetary policy is a function of the wealth of the unproductive agent. The second equation describes the marginal value for an unproductive agent holding $z$, so that $x=z$. The solution of this system, together with the boundary condition, fully characterizes the value of money in equilibrium.

Next we state a result that is key in characterizing the problem.

Lemma 2. Assume $\mu(x)$ is continuous in $[0,1]$ and that the forcing terms on the right hand side of (13)-(14) have no singularities in $(0,1)$. Then, it holds that $h^{u}(z)<0$ for all $z \in(0,1)$ and $\lim _{z \rightarrow 0} h^{u}(z)=0$.

See Appendix B for a proof. ${ }^{8}$ The economic content of the proposition is that unproductive agents deplete their share of money holdings as long as they remain unproductive. Lemma 2 allows us to characterize some interesting features of the Lagrange multipliers, the marginal value of money $p(z)$ and $u(z)$, by representing their evolution in the corresponding phase diagram. To this end we define the sets $L_{p}$ and $L_{u}$ with elements $(U, P) \in\left(\mathbb{R}^{+}\right)^{2}$, which evolve as $z$ varies in $[0,1]: U$ and $P$ are given by

$$
L_{p}: \quad P=\frac{\lambda}{\rho+\lambda+\mu(1-z)} u(z), \quad \text { and } \quad L_{u}: \quad P=\frac{\rho+\lambda+\mu(z)}{\lambda} u(z),
$$

according to $z$ that moves in its domain. These geometrical loci are very useful for investigating some features of the Lagrange multipliers. Indeed, by construction, $p^{\prime}(z) h^{p}(z)$ and $u^{\prime}(z) h^{u}(z)$ are zero on $L_{p}$ and $L_{u}$, respectively; thus, according to the sign of terms $h^{p}(z)$ and $h^{u}(z)$, both $L_{p}$ and $L_{u}$ determine different regions in the phase plane $(U, P)$ where the functions $u(z)$, $p(z)$ change behavior according to the sign of their derivative. Two examples are shown in the top panels of Fig. 1 where the arrows describe the increasing/decreasing behavior for $u(z)$

\footnotetext{
8 This result arises from the requirement of continuity and uniform Lipschitz condition in the space $(0,1)$ for the forcing terms of equations (13)-(14).
} 


\section{Phase diagrams}

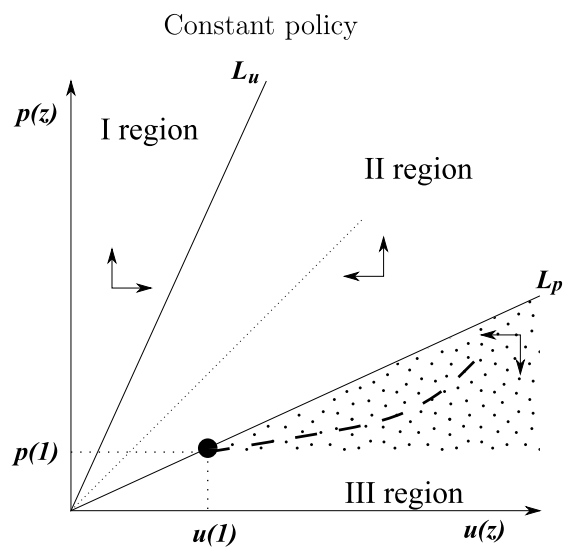

State dependent policy

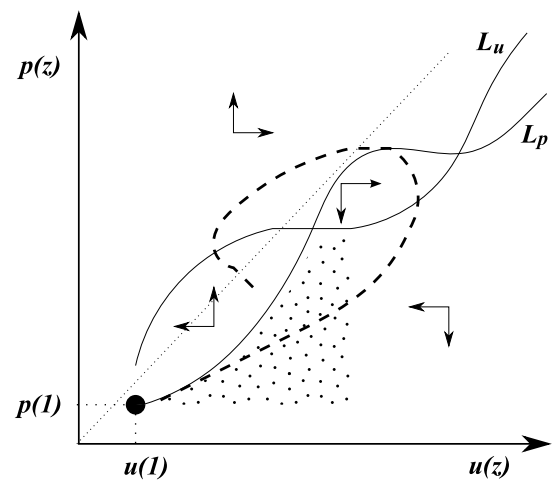

The shadow value of money: Lagrange multipliers
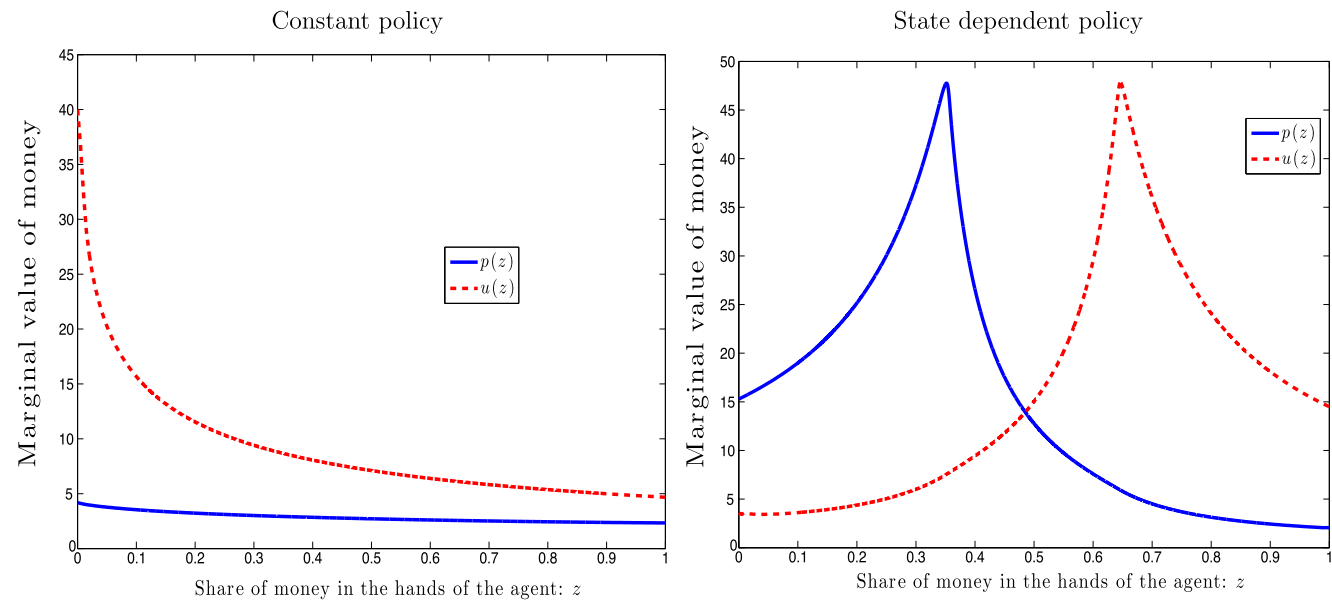

Fig. 1. The marginal value of money.

and $p(z)$. The direction of the arrows is determined by taking into account both the sign of terms $p^{\prime}(z) h^{p}(z), u^{\prime}(z) h^{u}(z)$ and the evolution of money holdings established in Lemma 2 . The phase diagrams describe the model dynamics: the first case, plotted in the upper-left panel, describes a constant money rule; the second case, describing a state-dependent rule, is plotted in the upperright panel. The bold dot in each plot corresponds to the boundary condition in equation (12), $u(1)=\frac{\rho+\lambda+\mu(0)}{\lambda} p(1)$, which lies on $L_{p}$, the 45-degree line $P=U$ is denoted by dotted line. The dashed curve is a possible path for the solution. Next we exploit the phase diagram to investigate some features of the solution under the different type of policies. In particular, our analysis allows us to provide global results for the constant policy. However, when the policy is allowed to vary with the state, the same results can be obtained only locally when $x$ is sufficiently small (i.e., when the productive agent holds most of the money).

When $\mu$ is constant, so that $\mu(x)=\mu \geq 0$ for every value of the aggregate state $x$, our results extend those provided in (Scheinkman and Weiss, 1986), which only considered the case where 
$\mu=0$. The phase diagram determines three regions where the functions $u(z)$ and $p(z)$ change behavior according to their derivative sign. It is evident that the only positive solutions of the problem must stay in the third region, where both functions are decreasing on the entire $(0,1)$ interval. More precisely, their path develops in the area that is dotted in the figure. The next proposition characterizes the marginal value of money when $\mu$ is constant.

Proposition 1. Under a constant policy $\mu(x)=\mu \geq 0$ we have that:

(i) $p(z)<u(z)$, and (ii) $p^{\prime}(z)<0, u^{\prime}(z)<0$ for all $z \in(0,1)$.

See Appendix B.4 for a proof. The first part of the proposition establishes that when the monetary policy is constant, the value of money for an unproductive agent is higher than the value of money for a productive agent, $u(z)>p(z)$, at all levels of money holdings $z \in(0,1)$. This property, first highlighted by Scheinkman and Weiss (1986), seems intuitive: Because the only difference between productive and unproductive agents holding $z$ is that the productive agent can work, an unproductive agent values more an extra unit of money; as a result, her Lagrange multiplier is higher. Second, the proposition also states that the functions $u(z)$ and $p(z)$ are decreasing in $z$ at all levels of money holdings $z \in(0,1)$. The bottom-left panel in Fig. 1 displays these properties and the upper-left panel presents the phase diagram explaining their origin: Since region III is the only admissible region for a solution to satisfy the boundary condition, the curves satisfy the properties listed in Proposition 1.

When the monetary policy depends on the state $x$, some interesting new features arise. Inspection of the upper-right panel of Fig. 1 shows that the equilibrium Lagrange multipliers must reach the boundary condition, which occurs when $x=0$ and the productive agent money holdings are $z=1$ (i.e., the bold dot in the figure), in the region that is under $L_{p}$ and $L_{u}$ (i.e., the dotted region). As a result, when the productive agent money holdings $z$ are large enough, the solution path develops in the area where both $p(z)$ and $u(z)$ are decreasing functions, as was the case for the constant policy. But crucially this result holds only locally, and in general the functions $p(z)$ and $u(z)$ that solve the problem can take many shapes. The next proposition states this result.

Proposition 2. Suppose that $\mu(x)$ is continuous and that the following assumptions hold:

$\left(A_{1}\right)$ the policy $\mu(x)$ satisfies $\frac{\lambda}{\rho+\lambda+\mu(0)}<\frac{\rho+\lambda+\mu(1)}{\lambda}$;

$\left(A_{2}\right)$ the policy $\mu(x)$ satisfies $\mu^{\prime}(x)<0$ for all $0 \leq x<v$, where $v \in \mathbb{R}^{+}$

Then there is a neighborhood for the productive agent money holdings at $z=1$, denoted by the interval $I_{z}$, where the multipliers satisfy

(i) $p(z)<u(z), \quad$ and (ii) $p^{\prime}(z)<0, u^{\prime}(z)<0$ for all $z \in I_{z}$.

See Appendix B.5 for a proof. The reason for the local similarity of the constant and the statedependent problem is the following. Start by noting that when the productive agent money holdings are $z=1$, the unproductive agent money holdings are $x=0$; in this case, by Lemma 1 , $\mu(0) \geq 0$. It follows by the continuity of $\mu(x)$ that $\mu(1-z) \geq 0$ for any $z \in I_{z}$. This nonnegativity constraint on the money growth rate, together with assumptions $A_{1}$ and $A_{2}$, produces a "local phase diagram," and implied behavior of the multipliers, that is similar to the behavior 
under a constant policy. As shown in the upper-right panel of Fig. 1, under the state dependent policy $\mu(x)$ we have that $u(z)>p(z)$ and that both curves are decreasing only when the unproductive agent money holdings are low (i.e., $x$ close to zero) and the productive agent money holdings are high (i.e., $z$ close to one). Assumptions $A_{1}$ and $A_{2}$ are important as they bound the local behavior of the dynamical system. Assumption $A_{1}$ guarantees that in the neighborhood of $z=1$ the locus $L_{p}$ lies below the locus $L_{u}$, as depicted in the upper-right panel of Fig. 1.9 Assumption $A_{2}$ guarantees that in the neighborhood of $z=1$, the locus $L_{p}$ is decreasing. While assumptions $A_{1}$ and $A_{2}$ are useful to analytically characterize the behavior of the economy under a state-dependent policy, they are not imposed in the numerical solution of the optimal policy that is developed below. We notice, however, that in all the cases that we analyzed they were satisfied at an optimum.

Notice that when $z \notin I_{z}$, the money growth $\mu(1-z)$ can be negative so that $u(z)$ can be below $p(z)$ and one (or both) of them can be increasing. To understand this result it is useful to contrast the state dependent case with the constant case. In the constant case the only difference between productive and unproductive agents is the production opportunity since policy is constant; as shown in Proposition 1 this immediately implies that $u(z)>p(z)$ for all $z$. But when the monetary rule varies with the state $x$, a comparative static across productivity states, i.e. comparison of $u(z)$ vs. $p(z)$, also involves a different path for the monetary rule. To make this point clear we make explicit the dependence of the marginal value of money on the money rule $\mu$. That is, let $\{p(z ; \mu(x)), u(z ; \mu(x))\}$ denote the marginal value of money for an agent with money holdings $z$ and where the money rule is $\mu$. Consider a productive agent holding money $z$. Under a constant policy her current value is $p(z ; \mu)$ and, if the state switches, her value will be $u(z ; \mu)$. Under the state dependent rule her current value is $p(z ; \mu(1-z))$ and, if there is a state switch, her value would become $u(z ; \mu(z))$. This shows that when the policy is state dependent the value of money across agent types differs not only because of differences in production opportunities, but also because the money rule depends on the wealth distribution. It follows that the restriction $p(z)<u(z)$ needs not to hold over the whole state space under a state-dependent rule. This feature, as we discuss next, allows for a substantial welfare improvement.

To understand why relaxing the properties (i) and (ii) of Proposition 1 is important for our problem, notice that the complete markets allocation features a constant consumption for all agents (see Remark 1). If a money rule existed to implement a first best allocation, it can be seen, using the first order conditions, that the rule would imply

$$
u(z)=p(1-z) \quad \text { for all } z \in[0,1]
$$

or, in words, that the functions $u(z), p(z)$ are symmetric around $z=1 / 2$. Obviously this feature cannot be achieved by a constant rule, since in that case the functions $u(z), p(z)$ are decreasing. The next proposition shows that the complete markets allocation cannot be sustained even under a state dependent policy (see B.6 for the proof).

Proposition 3. Let $\rho / \lambda>\bar{\rho}>0$. There is no monetary rule $\mu(x)$ that supports the complete markets allocation as a monetary equilibrium.

In spite of the impossibility to implement the first best, the state dependent monetary policy when $\mu$ is allowed to depend on $x$, is able, at least partially, to produce the required symmetry. This

\footnotetext{
9 To see this notice that this assumption can be extended to the inequality $\frac{\lambda}{\rho+\lambda+\mu(1-z)} \leq \frac{\rho+\lambda+\mu(z)}{\lambda}$ so that $L_{p}$ lies below $L_{u}$. A sufficient condition for satisfying this assumption is $\mu(1) \geq-\rho$.
} 
can be seen in the lower-right panel of Fig. 1 where we plot $u(z)$ and $p(z)$ under the optimal money rule $\hat{\mu}(x)$ computed in the next section. ${ }^{10}$

\section{The optimal regulation of the money supply}

In this section we use a standard ex-ante utilitarian welfare criterion to characterize the optimal regulation of money, namely a money supply rule $\mu(x)$ defined for $x \in[0,1]$. This amounts to solving a Ramsey problem: picking the best competitive equilibria, as defined in Section 3, under the assumption that there is commitment to the money supply rule $\mu(x)$.

Let $V\left(x_{0} ; \mu(x)\right)$ denote the discounted present value of the sum of utilities of both types of agents (who are given identical Pareto weights) for a given function $\mu(x)$ when the current state is $x_{0}$. The continuous time Bellman equation is

$$
\begin{aligned}
\rho V\left(x_{0} ; \mu(x)\right)= & \ln c^{u}\left(x_{0} ; \mu(x)\right)-c^{u}\left(x_{0} ; \mu(x)\right) \\
& +V_{x}\left(x_{0} ; \mu(x)\right) h^{u}\left(x_{0} ; \mu(x)\right)+\lambda\left(V\left(1-x_{0} ; \mu(x)\right)-V\left(x_{0} ; \mu(x)\right)\right) .
\end{aligned}
$$

where the notation emphasizes that consumption when unproductive $c^{u}\left(x_{0} ; \mu(x)\right)$, the dynamics of money holdings $h^{u}\left(x_{0} ; \mu(x)\right)$ and hence the value function $V\left(x_{0} ; \mu(x)\right)$ all depend on the money supply rule. As usual, the equation states that the flow value $\rho V$ equals the sum of the period utility for both agents plus the expected change in the value function. The latter occurs because of the evolution of money holdings (the change in $x_{0}$ ) as well as of the possibility that the identity of the productive agent will change. Notice that in this case the state, i.e., the wealth of the unproductive agent, switches from $x_{0}$ to $1-x_{0}$.

The planner's problem is to find the best money supply function $\mu(x)$, in the sense that

$$
v\left(x_{o}\right)=\max _{\mu(x)} V\left(x_{o} ; \mu(x)\right)
$$

We consider the problem from an ex-ante perspective, i.e., assuming that at the "beginning of time" nature assigns the initial productive states and the planner can choose the initial wealth distribution and a policy rule for money growth. We assume that the planner can commit to the policy rule. Note that because individual types are not observable, and given the symmetry of the environment (and identical Pareto weights), the planner will give the same amount of liquidity to every agent and therefore it is ex-ante optimal to set $x_{0}=\frac{1}{2}$. Thus the planner chooses the function $\mu(x)$ in order to maximize $v(1 / 2)$.

To evaluate the policy it is useful to define a certainty equivalent compensating variation. Let $\alpha$ denote the consumption equivalent cost of market incompleteness associated with a given policy. That is, $\alpha$ solves the following equation

$$
2 \ln (1-\alpha)-2=\rho v(1 / 2)
$$

so that $\alpha$ measures the fraction of the consumption under complete markets that agents would be willing to forego to eliminate the volatility of consumption due to market incompleteness for a given policy rule $\mu .^{11}$

Having established that the complete markets allocation cannot be achieved (Proposition 3), we look for the optimal policy $\hat{\mu}(x)$ by searching numerically for the policy that maximizes the

\footnotetext{
10 The optimal policy $\hat{\mu}(x)$ satisfies assumptions $A_{1}$ and $A_{2}$ so that $u(z)$ and $p(z)$ satisfy Proposition 2 .

11 Recall that under complete markets $c^{u}(z)=c^{p}(z)=1$ for all $z$, and $l^{p}(z)=2$ for all $z$.
} 
ex-ante expected welfare $v(1 / 2)$. Since the system of differential equations that characterizes the equilibrium allocations cannot be solved in closed form we must resort to numerical analysis. We solve the model by using a standard value function iteration approach, to compute equation (15), and a discretization method to solve the system of differential equations in equations (13)-(14) with the associated boundary conditions in equations (11)-(12). It is worth mentioning a numerical difficulty that arises as $x \rightarrow 0$ due to the fact that $h^{u}(x) \rightarrow 0$. This implies that the forcing term in equation (14) may diverge and potentially generate an error propagation issue. We tackle this issue by an appropriate grid choice for the evaluation routine. ${ }^{12}$ We restrict our attention to monetary policies that are piecewise linear continuous. ${ }^{13}$ For simplicity the results discussed in this section are obtained by considering a policy function with five nodes. In Appendix D we show that the optimal policy $\hat{\mu}(x)$ shares the same properties if we allow for more nodes, and the welfare gains for allowing more nodes are small. ${ }^{14}$ It is also worth mentioning that neither assumption $A_{1}$ or $A_{2}$ are imposed in the algorithm used to solve the problem. Details on the numerical solution algorithm can be found in Appendix C. To aid in the understanding of the policy we also compute an alternative policy, $\bar{\mu}$, which maximizes $v(1 / 2)$ under the restriction that the policy has to be constant. Fig. 2 plots both policies obtained for the baseline parametrization of the model where the normalized discount rate $\rho / \lambda$ equals $1 / 2$. Given this parameter choice, setting the discount rate to the standard value of 0.05 implies that $\lambda$ equals $1 / 10$, so that the average length of a productive state is 10 periods. Qualitatively similar results are obtained for other parameterizations for $\rho / \lambda$ (see below).

The constant policy $\bar{\mu}$ consists of an expansion of the monetary base of 0.1 percent; $\bar{\mu}$ is positive because, for $\rho / \lambda=1 / 2$, the insurance motives outweigh the inflation costs. ${ }^{15}$ The problem solved by the constant policy is reminiscent of the problem solved by Imrohoroglu (1992) (see the related literature survey in the online Appendix for a discussion of the relation between these papers). As shown in Fig. 2 the optimal state dependent policy $\hat{\mu}(x)$ is very different: it prescribes an expansion of the money supply when $x$ is low and a contraction of the money supply when $x$ is high. This happens because the optimal policy is able to decouple the insurance motives and the provision of incentives (a high return on money) through its state-dependent nature. The welfare cost of market incompleteness $\alpha$, as defined in equation (17), under the constant policy is 31.7 percent while under the optimal policy is 3.3 percent, which shows that the optimal policy increases welfare substantially. Finally notice that, although the state dependent policy is continuous in the state variable $x$, the policy is discontinuous whenever the productive state switches from $x$ to $1-x$ : in these circumstances the policy jumps from $\mu(x)$ to $\mu(1-x)$.

\footnotetext{
12 For instance the forcing term diverges as $x \rightarrow 0$ in the original Scheinkman and Weiss formulation where $\mu$ is constant and equal to zero. More details on the numerical approach are given in Appendix C.1.

13 Although we do not provide a formal proof, we argue that the optimal policy function $\mu(x)$ is continuous in $x$ as a consequence of the Theorem of the Maximum (see Stokey and Lucas, 1989, p. 62). In order to satisfy the theorem, given that the period utility function is continuous, all we need is that the correspondence $\Gamma: S \rightarrow S^{\prime}$ is continuous, which holds in our setting. This follows as the equilibrium requires that, for any policy rule, $\mu(0)>0$, which bounds the marginal values of money $u$ and $p$.

14 We also explored solving the problem using smooth (differentiable) functions, such as quadratic B-splines, which produced similar results.

15 The online Appendix explores how $\bar{\mu}$ changes with $\rho / \lambda$. For low values for $\rho / \lambda$, when the inflation costs outweigh the insurance motives, $\bar{\mu}=0$. For larger values for $\rho / \lambda, \bar{\mu}$ exhibit a monotonic relationship with the normalized discount rate; this is a natural consequence of the insurance motives being increasing in $\rho / \lambda$.
} 


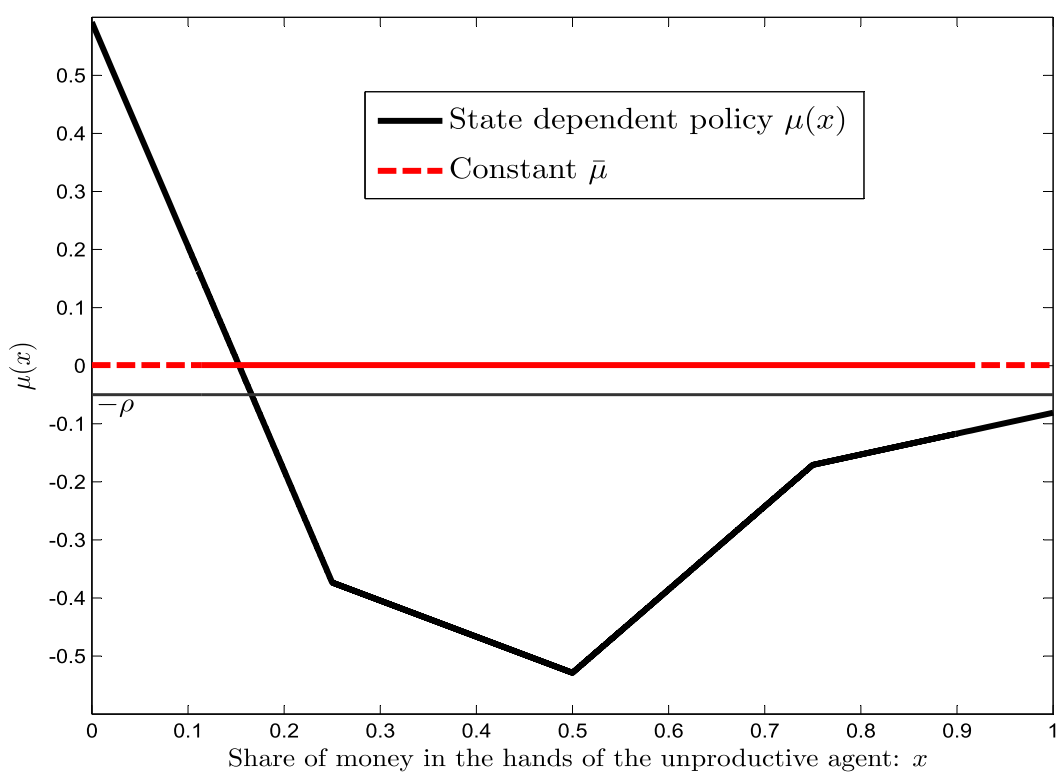

Parameter: $\rho / \lambda=0.5$.

Fig. 2. Optimal policy.

We now describe the features of the optimal policy and interpret them in terms of the trade-off between the provision of insurance versus the provisions of incentives to produce (by offering a high expected return on money to the productive agent). When the insurance motives are very strong, which happens when $x \approx 0$, the policy prescribes the largest expansion of the money supply. As the insurance motives decrease, which happens as $x$ moves away from zero, the optimal money growth rate falls. Eventually, at $x \approx 0.2$, the motive for providing production incentives outweighs the insurance role of monetary policy and, as a result, the policy turns to prescribing monetary contractions. When $x$ lies in an intermediate region the policy prescribes the largest monetary contractions, much below the money growth rate associated with the Friedman rule in deterministic environments. ${ }^{16}$ These extreme contractions are a reflection of the large expansions that occur when $x$ is low: because expansions damage the return of money, large (future) monetary contractions undo the detrimental effects of the expansions on production incentives (as agents are forward looking). Intuitively, the expansions and contractions compensate each other, striking a balance between the insurance provision and the production incentives. As $x$ increases towards 1 the level of the monetary contractions decreases. This is a reflection of the provision of insurance that might be needed if a switch of the state occurred: when $x$ is high the unproductive agent is rich, and her insurance needs are small. But the productive agent is poor and, if a state switch occurred, she would be unproductive and with a high insurance need. This would then trigger a large monetary expansion. Since monetary contractions are regressive when $x>1 / 2$ (i.e. they tend to increase $x$, all else the same), contractions become less attractive at high levels of $x$. This explains the v-shaped form of the optimal policy.

16 The Friedman rule advocates that the rate of return should equal the time discount. In many deterministic models the rule can be implemented by contracting the money supply at the rate of time discount. 


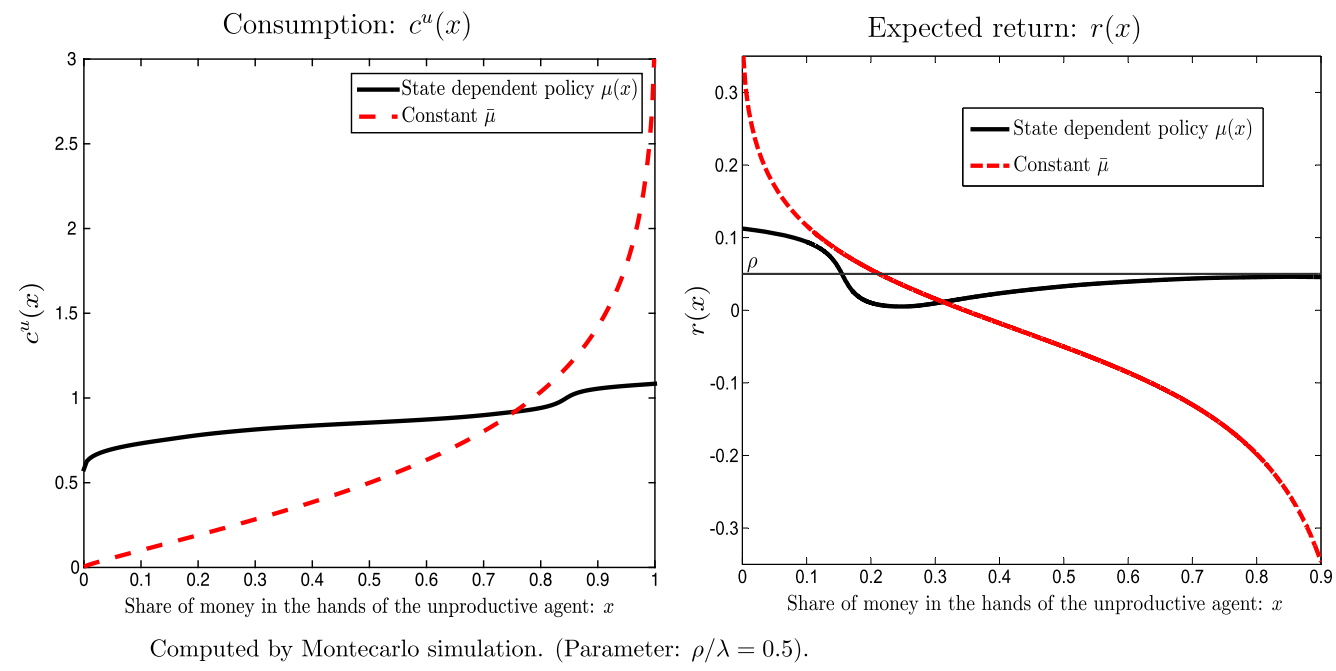

Fig. 3. Consumption when unproductive and the return on money.

Fig. 3 illustrates the profiles of the consumption function and the expected return on money under the optimal policy $\hat{\mu}(x)$ and the constant policy $\bar{\mu} .{ }^{17}$ The main result, illustrated in the left panel of the figure, shows that the consumption of the unproductive type under the optimal policy is (i) smoother than under the constant policy, and (ii) closer to the consumption implied by the complete markets allocation (where $c^{u}(z)=1$ ). The smoother consumption profiles of the state-dependent rule also yields a flatter profile for the expected return on money $r(x)$, as shown in the right panel of the figure. The right panel of Fig. 3 shows that the expected return on money is high when $x$ is low. The expected return on money is proportional to the consumption growth of the productive agent. At $x=0$ the productive agent is consuming $c=1$, and expects, in case of a state switch, to consume $c^{u}(1)>1$, which explains the high expected return at $x=0$. The same logic explains the expected low return on money when production is high (i.e. when $x$ is high). The figure shows that the less extreme values of consumption (under the state dependent policy) translate into less extreme values of the expected returns of money. ${ }^{18}$

To aid in the analysis of the optimal policy, we results obtained by numerical simulations of the model. The simulations are simple to compute given the functions $u(z)$ and $p(z)$ for the marginal values of money and the money growth rate. We stress that these functions, produced by solving the planner's problem, completely determine all equilibrium objects of the economy. The simulation simply involve a sequence of state switches, that occur with Poisson rate $\lambda$, which immediately yield the time series for $x$, and for any other economy outcome such as the consumption level, or the real rate of return of money. Fig. 4 plots the times series for the consumption of one agent over one particular sample path, produced by a simulation. The upper panel describes the sample path under the state dependent rule, the bottom panel under the constant rule. The sample realization of productivity switches is common across the two panels: it can be seen that when the periods in which consumption is constant at 1 (i.e. when the agent is productive) are the

17 See Appendix A for details on derivation of the expected return of money.

18 The price of money $q(x)$ can be seen in the lower-right panel of Fig. 1 by noticing that $q(x)=p(1-x)$. 

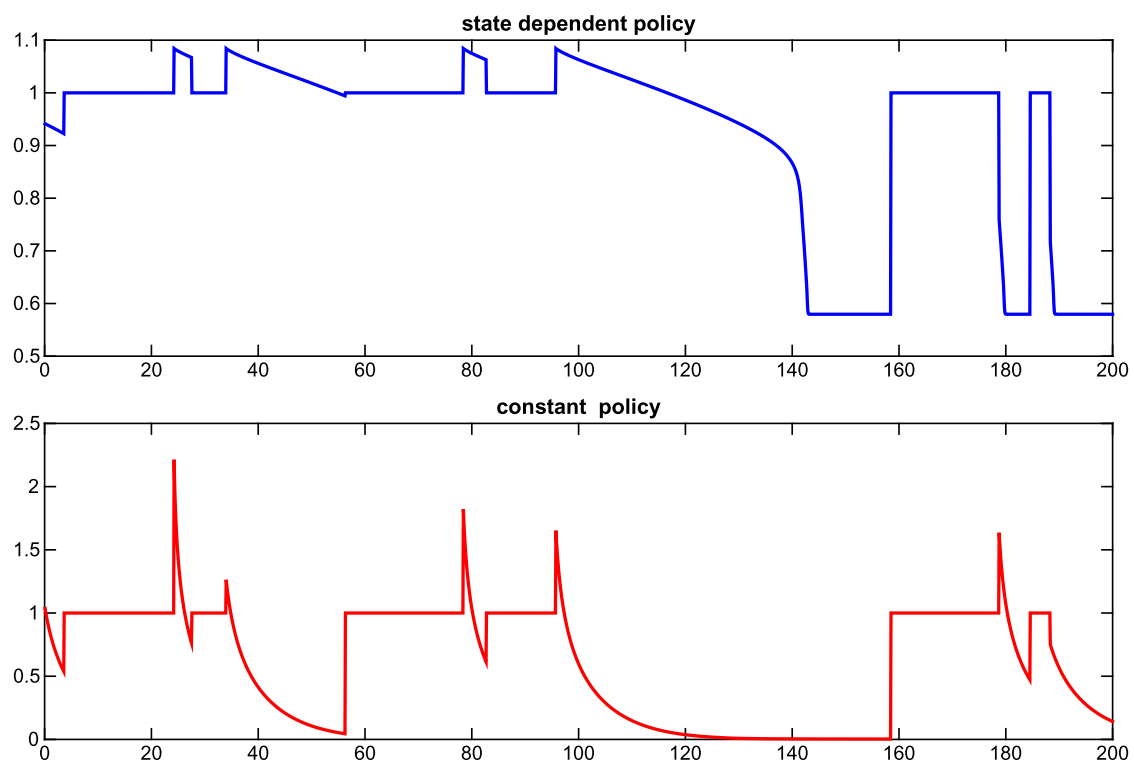

Produced by a Monte Carlo simulation with Parameter: $\rho / \lambda=1 / 2$.

Fig. 4. Sample paths for the consumption of Agent 1.

Table 1

Economic outcomes under alternative policy rules: Summary statistics.

\begin{tabular}{lll}
\hline & State dependent policy, $\hat{\mu}(x)$ & Constant policy, $\bar{\mu}$ \\
\hline $\mathbb{E}[\mu]$ & 0.22 & 0.001 \\
$\operatorname{Std}[\mu]$ & 0.34 & 0 \\
$\mathbb{E}[r(x)]$ & 0.08 & 0.05 \\
$\operatorname{Std}[r(x)]$ & 0.03 & 0.18 \\
$\mathbb{E}\left[c^{u}(x)\right]$ & 0.8 & 0.3 \\
$\operatorname{Std}\left[c^{u}(x)\right]$ & 0.2 & 0.4 \\
Fraction of time $x<0.025$ & 0.48 & 0.13 \\
\hline
\end{tabular}

Computed by Montecarlo simulation. (Parameter: $\rho / \lambda=0.5$ ).

same in the two panels. But it is apparent that consumption is smoother and on average higher under the state dependent policy.

Table 1 reports the mean and standard deviation for some statistics of interest under the invariant distribution, both under the best constant policy and under the best state dependent policy. As the table shows, the unproductive agent spends much more time being poor (with a ow $x$ ) under the state dependent policy. This is the consequence of the optimal insurance provided by the state dependent policy which alters the welfare consequences of ending up with low wealth. In spite of the fact that the unproductive agent spends more time with $x \approx 0$ under the state dependent policy, the unproductive agent enjoys higher average consumption under the state dependent policy than under a constant policy ( 0.8 vs 0.3$)$ and a lower variability of consumption (the standard deviation is 0.2 under state dependent policy vs. 0.4 under constant policy). 

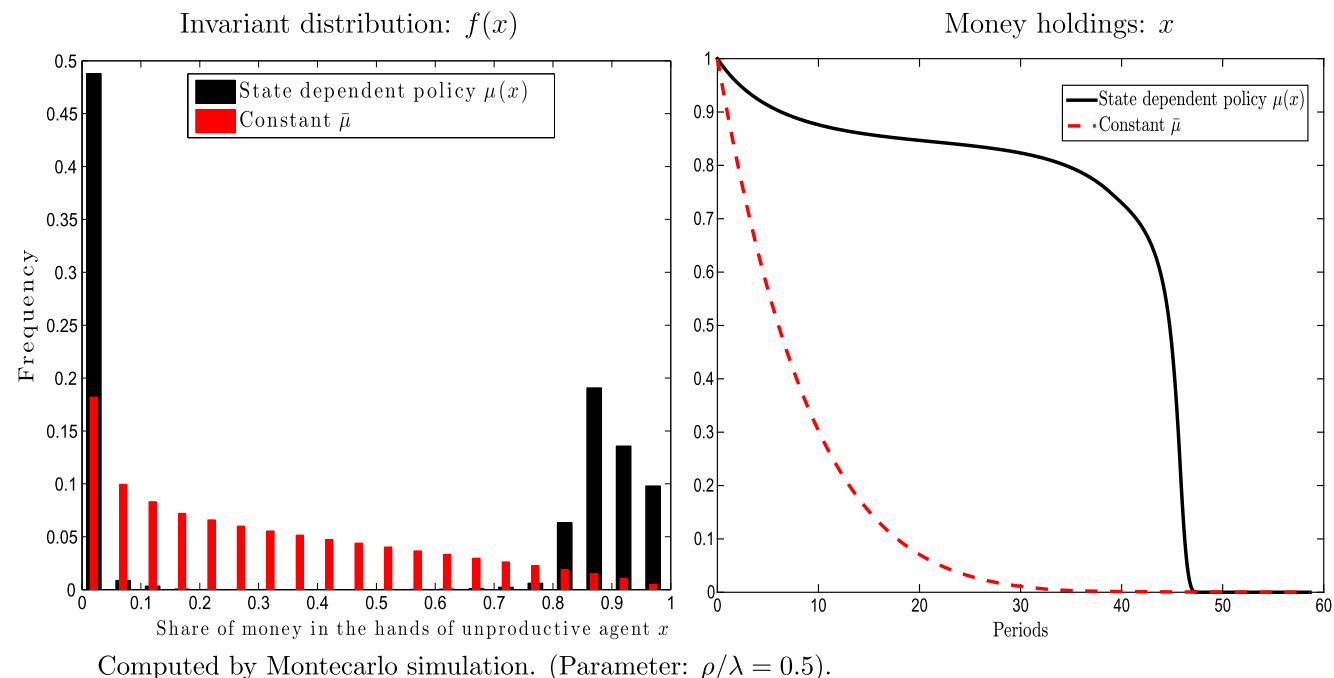

Computed by Montecarlo simulation. (Parameter: $\rho / \lambda=0.5$ ).

Fig. 5. Wealth distribution and Money holding behavior.

The left panel of Fig. 5 shows the histograms of the invariant distribution of the unproductive agent's wealth under the best constant policy (where $\mu=0.001$ ) and the best state-dependent policy of Fig. 2. As mentioned, the unproductive agents spends more time near $x \approx 0$ under the state dependent policy than under the constant policy. This is an immediate consequence of the insurance provision. However the consumption profile is overall smoother and higher (on average) under the state dependent policy as can be seen from Fig. 3. The right panel shows the dynamics of the money demand holdings for an unproductive agent as a function of the initial state $x$ (vertical axis) and the time elapsed being unproductive (horizontal axis). The figure shows how wealth is decumulated over time (under the assumption that productivity does not change). It can be seen that the unproductive agent escapes from high wealth much faster under the constant policy (a fact that is reflected in the low mass over high values of $x$ in the histogram of the left panel). The mirror image of this pattern is that under the constant policy the unproductive agent avoids going near $x \approx 0$ because that means low consumption, while the agent dives towards zero very quickly under the state dependent policy as soon as $x$ gets low and the transfers prescribed by the state dependent rule materialize.

Notice that the state-dependent pattern of the policy rule can be equivalently interpreted in terms of the business cycle. As aggregate production is increasing in $x$ (because consumption of the unproductive agent is increasing in $x$ - see the left panel of Fig. 3), then the optimal policy $\hat{\mu}(x)$ is such that monetary expansions happen when aggregate production is low and monetary contractions occur otherwise. In other words, the policy is expansionary during recessions and contractionary during expansions. Furthermore, the fact that there is an invertible mapping between the wealth level $x$ and aggregate production implies that the requirement that the planner observes $x$ can be equivalently understood as requiring the planner to observe aggregate production or some other aggregate variable such as the price level.

The model also produces a novel mechanism to interpret high frequency changes in the velocity of money (the ratio between GDP and real balances), namely the fact that the money growth rate is not equal to inflation at every point in time. This is immediately seen in the right panel of Fig. 3 by noting that even in the case with constant money $\bar{\mu}$ we have that inflation (related 


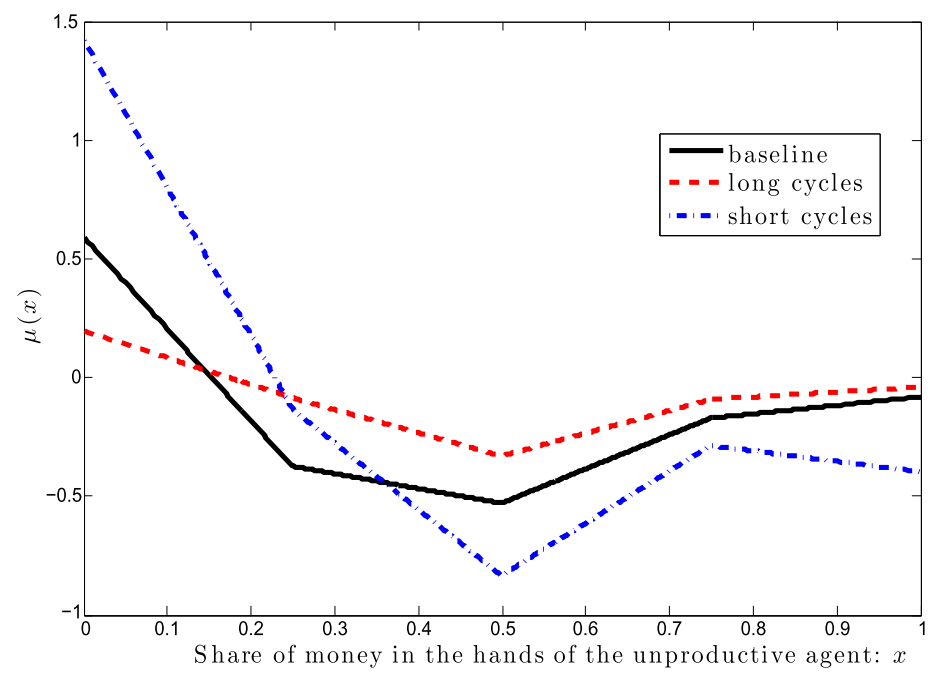

Note on parameters: baseline uses $\rho / \lambda=0.5$, long cycles uses $\rho / \lambda=1$, short cycles uses $\rho / \lambda=0.1$.

Fig. 6. Optimal policy.

to the inverse of the return on money) is not constant. The high frequency data on money and inflation produced by this model will not align on the 45 degree line, even though in the long run (e.g. averaging over very many periods) inflation is equal to money growth. The mechanism behind this fact is that the precautionary demand for money varies along the state of the cycle (as reflected in the time varying price of money).

To conclude this section we explore the effect of changing the fundamental parameter of the model: $\rho / \lambda$. This is a "normalized" discount factor, i.e. the ratio between the intertemporal discount rate $\rho$ and the arrival rate of productivity switches $\lambda$. Low values of $\rho / \lambda$ describe an economy in which the agents are patient (low $\rho$ ), or equivalently the expected length of a productive cycle is short (high $\lambda$ ). In Fig. 6 we plot the optimal policy under three different parametrizations. The first case uses our baseline parameter values, $\rho / \lambda=0.5$; the second case uses $\rho / \lambda=1$ which represents an economy with a more impatient agent (relative to the baseline) or equivalently an economy with longer productive cycles. The third ${ }^{\circ}$ case, $\rho / \lambda=0.1$, is an economy with more patient agents (relative to the baseline) and/or shorter cycles. As the figure shows, the qualitative features of the policy are similar for the three parameter configurations: high monetary injections when insurance motives are high (when $x$ is low), and high monetary contractions when they are the lowest (when $x$ is close to 1/2). Even though similar in qualitative terms, these cases differ in the level of monetary injections. Monetary expansions and contractions are more extreme when $\rho / \lambda$ is small. Our interpretation of these results is that shorter cycles, and/or more patient agents, insurance is easier to provide since the monetary expansions is going to be short lived (cycles are short) and/or agents are more patient which allows a more efficient substitution of the returns across time (i.e. current expansions are more easily compensated by future contractions).

Finally, as previously discussed, the welfare $\operatorname{cost} \alpha$, which is 3.3 percent under the baseline configuration, increases to 11.7 percent when the cycle is long (high $\rho / \lambda$ ), and falls to 0.3 percent 
when the cycle is short ( small $\rho / \lambda$ ). The improvement in welfare as the cycle shortens follows because the insurance motives decrease as the average length of an unproductive spell falls.

\section{Concluding remarks}

In a variety of models monetary policy has persistent effects on the distribution of wealth (money or other assets). These distributional effects are often muted or kept to a minimum in theoretical models to preserve tractability. This paper explored how these distributional concerns affect the optimal monetary policy in a monetary model that allows for a full dynamic analysis of the (two-way) interactions between monetary policy and the wealth distribution.

Two forces shape the design of optimal policy in this setup. As in other monetary models, distortions are minimized when the return on money equals the rate of time discount (i.e., Friedman's rule). However with uncertainty and incomplete markets an expansionary policy can be desirable due to insurance needs. A trade-off arises as expanding the liquidity base dampens the return on the asset, therefore reducing the production incentives. The optimal regulation of the money supply strikes a balance between these two forces. Since the relevance of these forces depends on the wealth distribution, which evolves through the business cycle, the optimal (anticipated) monetary policy is state dependent.

The novelty of our paper is that we acknowledge this dependence and we explore how the state-dependent policy balances the costs of anticipated inflation with the needs for insurance along the business cycle. This policy allows for a dramatic improvement in welfare compared with a policy that does not respond to the state. The optimal policy expands the supply of liquidity when the unproductive agents are poor (when the insurance needs are large), and it contracts the liquidity base otherwise to maximize production incentives. The principle underlying this prescription is due to the state-dependent redistributive role of monetary policy, and it differs from the one arising in sticky-price models. Because aggregate production is low when the unproductive group is poor and high when they are rich, the best policy can be interpreted as counter-cyclical. Interestingly, in spite of the policy being far away from contracting the money supply at the rate of preference, the optimal policy "echoes" Friedman's rule as the expected real return of money approaches the rate of preference.

While the specific predictions of the analysis likely depend on the details of the model, the paper highlights the potential relevance of a transmission channel of monetary policy that seems reasonable and little explored. Several interesting extensions are left for future work. An important, and classical, assumption for our results is that the planner has the ability to levy lump-sum taxes, and that agents are not allowed to renege from their obligations. However, as pointed out by Andolfatto (2013), if agents are allowed to voluntarily be subject to taxation, the degree of monetary contractions has to be limited by the voluntary nature of participation. Therefore, it is possible that the incentive-feasible allocation cannot support the optimal policy we constructed in this paper, as this one requires large monetary contractions. An interesting open question is to use a mechanism design approach to find the optimal state-dependent policy under voluntary participation. Another interesting extension is to reformulate our model, following Atkeson and Lucas (1992), considering an endowment economy where agents are subject to preference shocks. If only two types of agents are considered, as we did in this paper, the two setups share several similarities and the solution developed for this paper can also be used in this other context. 


\section{Appendix A. The expected return on money}

The expected return on money, $r(x)$. Define the stochastic expected net return on money for a small time interval $\Delta$ as

$$
r(x) \Delta=\mathbb{E}\left[\frac{\tilde{q}_{t+\Delta}}{\tilde{q}_{t}}-1 \mid x_{t}=x\right]=\mathbb{E}\left[\frac{q_{t+\Delta}}{q_{t}}-\Delta \mu_{t}-1 \mid x_{t}=x\right],
$$

where $\tilde{q}_{t}=q\left(x_{t}\right) / M_{t}{ }^{19}$ Without loss of generality consider the case where at time $t$ agent 1 is productive with money holdings given by $z_{t}^{1}$, and let $z_{t}^{2}$ denote the money holdings of the unproductive agent at time $t$. Then, using that $q(x)=p(z)$, we have

$$
r\left(x_{t}\right) \Delta=\frac{(1-\lambda \Delta) p\left(z_{t+\Delta}^{1}\right)+\lambda \Delta p\left(z_{t+\Delta}^{2}\right)}{p\left(z_{t}^{1}\right)}-1-\Delta \mu_{t} .
$$

A first order Taylor expansion of $p\left(z_{t+\Delta}^{i}\right)$ gives

$$
\begin{aligned}
r\left(x_{t}\right) \Delta= & \frac{p^{\prime}\left(z_{t}^{1}\right) h^{p}\left(z_{t}^{1}\right) \Delta}{p\left(z_{t}^{1}\right)}+\lambda \Delta\left(\frac{p\left(z_{t}^{2}\right)+p^{\prime}\left(z_{t}^{2}\right) h^{p}\left(z_{t}^{2}\right) \Delta}{p\left(z_{t}^{1}\right)}-\frac{p\left(z_{t}^{1}\right)+p^{\prime}\left(z_{t}^{1}\right) h^{p}\left(z_{t}^{1}\right) \Delta}{p\left(z_{t}^{1}\right)}\right) \\
& -\Delta \mu_{t} .
\end{aligned}
$$

Then, taking the limit of $r\left(x_{t}\right) \Delta / \Delta$ as $\Delta \rightarrow 0$ gives $r\left(x_{t}\right)=\frac{p^{\prime}\left(z_{t}^{1}\right) h^{p}\left(z_{t}^{1}\right)}{p\left(z_{t}^{1}\right)}+\lambda\left(\frac{p\left(z_{t}^{2}\right)}{p\left(z_{t}^{1}\right)}-1\right)-\mu_{t}$. Using equation (9) gives $r\left(x_{t}\right)=\rho+\lambda\left(\frac{p\left(z_{t}^{2}\right)}{p\left(z_{t}^{1}\right)}-\frac{u\left(z_{t}^{1}\right)}{p\left(z_{t}^{1}\right)}\right)$ or, using that in this proof we assumed that agent 2 was unproductive at time $t$, i.e. $x_{t}=z_{t}^{2}$.

$$
r(x)=\rho+\lambda \frac{p(x)-u(1-x)}{p(1-x)}=\rho+\lambda \frac{p(x)}{p(1-x)} \frac{c^{u}(1-x)-1}{c^{u}(1-x)} .
$$

In a complete markets setting, such as the one described in Remark 2, consumption is constant and the expected real return equals the time discount, $\rho$. With incomplete markets the return on money depends on the history of the shocks, as summarized by the wealth distribution $x$.

\section{Appendix B. Proofs}

\section{B.1. Proof of Remark 2}

Let $i=1$ be the index for the unproductive agent, and consider her decision problem. The money supply growth is $\mu=\frac{\dot{M}_{t}}{M_{t}}$ and let $\tilde{u}_{t}$ denote the Lagrange multiplier of the money flow constraint in equation (3). The first order condition with respect to $c_{t}$ gives: $u_{t}=q_{t} / c_{t}$, where we used the homogeneity of degree -1 in the aggregate money supply $M_{t}$ for both $\tilde{u}_{t}$ and $\tilde{q}_{t}$. The Euler equation for $\dot{m}_{t}^{1}$ gives $\rho=\frac{\dot{u}_{t}}{u_{t}}-\frac{\dot{M}_{t}}{M_{t}}=\frac{\dot{q}_{t}}{q_{t}}-\frac{\dot{c}_{t}}{c_{t}}-\mu$ where the last equality uses $u_{t}=q_{t} / c_{t}$. Notice that this is solved by $c_{t}=1, \dot{c}_{t}=\dot{q}_{t}=0$, and $\mu=-\rho$.

The constant level of $q$ is pinned down by imposing that total nominal assets at the beginning of a cycle, which in the stationary equilibrium are held by the unproductive agent, equal total nominal consumption plus tax receipts over the cycle of length $T$. Without loss of generality

19 Notice that, because of Jensen's inequality, the expected return on money does not coincide with the inverse of the expected inflation rate (see the online Appendix for a calculation). 
let's consider the first cycle, starting at time 0 , where the unproductive agent holds all the money supply; $m_{0}=M_{0}$. Using that $M_{t}=M_{0} e^{\mu t}, \mu=-\rho$, and $\tilde{q}_{t}=\frac{1}{M_{t}} q$, we have

$$
\int_{0}^{T} \frac{1}{\tilde{q}_{t}} d t+\int_{0}^{T} \rho M_{t} d t=M_{0}, \quad \text { which gives } \quad q=\frac{1-e^{-\rho T}}{\rho e^{-\rho T}} \cong \frac{T}{1-\rho T},
$$

where the approximation is accurate for small $T$. It is immediate to verify that this allocation also solves the Euler equation of the productive agent and that her money holdings are never negative.

\section{B.2. Proof of Lemma 1}

We first present a proof of the first part of the statement, namely that there is no monetary equilibrium if $\mu(x)<0 \forall x$. We then present a proof of the second part of Lemma 1 .

A contractionary policy $\mu(x)<0 \forall x$ requires agents to pay lump sum taxes $(\tau(x)<0 \forall x)$. Consider the case where agent 1 has fraction of money balances $z_{t}$, and the current state of the economy is $\mathcal{I}_{t}=0$, which means that agent 1 is unproductive. If $z_{t}$ is low enough, given that $\lambda>0$ and finite, the agent will fail to comply with the monetary authorities with non-zero probability. On the other hand, consider the case where $z_{t}=1$. In this case the agent is able to comply with her tax obligations with certainty, as she can make her consumption profile to be arbitrarily low. This implies that there exists a threshold $\zeta \in(0,1)$ such that for $z^{i} \geq \zeta$ the agent is able to cover her lifetime tax needs with probability one. Note that the threshold must be independent of the current state $\mathcal{I}_{t}$ as with positive probability the states are reversed. In the next claim we characterize this threshold.

Claim 1. If $\mu_{t}<0 \forall t$, for any state of the world $\mathcal{I}_{t}$, there is a unique threshold: $\zeta=1 / 2$, and a unique ergodic set where $z_{t}=\frac{1}{2} \forall t$, that ensures tax solvency.

Proof. We will first prove by contradiction that $\zeta \notin[0,1 / 2)$. Then we will show that $\zeta=1 / 2$ is enough to cover the lifetime tax obligations. Suppose that $\zeta<1 / 2$. Without loss of generality assume that $z_{t} \in(\zeta, 1 / 2)$ and agent 1 is unproductive. Conditional on no reversal of the state, it follows that $z_{t+\Delta}<z_{t}$ for any $\Delta>0$. Then for a given $\Delta \in \mathbb{R}^{+}, \operatorname{Pr}\left[z_{t+\Delta}<\zeta\right]>0$ and therefore the agent will fail to comply with her tax obligations with positive probability. Then, $\zeta \notin[0,1 / 2)$. Consider now the case where $z_{t}=\zeta=1 / 2$. As the agent can decide not to trade she can always keep her share of outstanding money balances $z$ above $1 / 2$ and therefore for any $\mu \in(0,1)$ she will be able to cover her tax needs. That $z=1 / 2$ is the ergodic set is trivial. If $z<1 / 2$ there is a positive probability that an agent fails to pay for her lifetime taxes. An unproductive agent with money holdings $z>1 / 2$ is willing to buy goods (and the productive one with $z<1 / 2$ willing to take the money) until $z$ reaches $1 / 2$.

Intuitively, given the uncertain duration of the productivity spell, the only value of money holdings that ensures compliance with tax obligations for both types of agents is $z=1 / 2$. At this point, for any history of shocks, the identical lump-sum (negative) transfers reduce the money holdings of both agents proportionally, leaving the wealth distribution unaffected. This leads us to

Remark 3. Let $\mu(x)<0 \forall x$ : In the ergodic set there is no stationary monetary equilibrium and consumption allocations are autarkic. 
The proof of Remark 3 follows from noting that Claim 1 implies no trade in the ergodic set. Productive agents have an unsatisfied demand for money and unproductive ones have an unsatisfied demand for consumption goods.

Now we turn to prove that $\mu(0) \geq 0$. Consider the law of motion for the share of money held by type 1 when unproductive, $h^{u}(z)=\mu(x)\left(\frac{1}{2}-z\right)-\frac{c^{u}(z)}{q(x)}$. Because $c^{u}(z) \geq 0$ and $q(x)>0$ (i.e. prices are positive in a monetary equilibrium) we can bound above the law of motion $h^{u}(z)$ : $h^{u}(z) \leq \mu(x)\left(\frac{1}{2}-z\right)$. Notice that because the agent is unproductive $z=x$. We apply this result into the previous equation and evaluate it at $x=0$ to get $h^{u}(0) \leq \frac{\mu(0)}{2}$, from where it can be seen that $h^{u}(0)<0$ if $\mu(0)<0$ which is inconsistent with the borrowing and tax-solvency constraints. Therefore, $\mu(0) \geq 0$.

\section{B.3. Proof of Lemma 2}

The boundary condition (11) yields $\lim _{z \rightarrow 0} h^{u}(z)=0$. Moreover, we have $h^{u}(1 / 2)=$ $-\frac{1}{u(1 / 2)}<0$. Under the continuity assumption on $\mu(x), h^{u}(z)$ is a continuous function; then the inequality can be extended over a suitable neighborhood of the asset $z=\frac{1}{2}$. More precisely, it is possible to consider an interval $\left(\frac{1}{2}-\delta, \frac{1}{2}+\delta\right) \subset(0,1)$ where $h^{u}(z)<0$. The further assumption related to the lack of singularities yields that the function $h^{u}(z)$ cannot nullify within the domain and its sign has to be uniform. It follows that the interval $\left(\frac{1}{2}-\delta, \frac{1}{2}+\delta\right)$ overlaps the whole integration interval and the previous inequality holds for all $z \in(0,1)$. In this way, the result is completely obtained.

The assumptions in the proposition mean that the solution $(u(z), p(z))$ evolves for $z \in(0,1)$ in a region of $\mathbb{R}_{+}^{2}$ where the forcing term has no singularity and is continuous, with the classical uniform Lipschitz condition locally satisfied. We recall that this kind of smoothness is a basic assumption which is needed for investigating the solution of the differential problem. As discussed in Ascher et al. (1988), smoothness together with the requirement of solvability of the algebraic system related to the boundary conditions are the crucial issues for establishing the existence of a solution.

\section{B.4. Proof of Proposition 1}

The proof follows from the inspection of the phase diagram, which is plotted in upper-left Fig. 1. We assume the policy $\mu(x)=\mu \geq 0$ is constant and exploit the geometric loci in $\left(\mathbb{R}^{+}\right)^{2}$ defined as

$$
\begin{array}{ll}
L_{p} & =\left\{(U, P) \in\left(\mathbb{R}^{+}\right)^{2} \mid P=\frac{\lambda}{\rho+\lambda+\mu} U\right\}, \quad \text { and } \\
L_{u} & =\left\{(U, P) \in\left(\mathbb{R}^{+}\right)^{2} \mid P=\frac{\rho+\lambda+\mu}{\lambda} U\right\} .
\end{array}
$$

We first note that $\frac{\lambda}{\rho+\lambda+\mu}<1<\frac{\rho+\lambda+\mu}{\lambda}$; this implies that the locus $L_{p}$ lies under $L_{u}$ and the dotted line $P=U$ is between them, as it is shown in the figure. Then, according to equations (13)-(14), the terms $p^{\prime}(z) h^{p}(z)$ and $u^{\prime}(z) h^{u}(z)$ are zero on $L_{p}$ and $L_{u}$, respectively. Thus, the uniform signs of $h^{u}(z)<0$ and $h^{p}(z)>0$ allow us to gain an insight about the increasing/decreasing pattern of the Lagrange multipliers $p(z)$ and $u(z)$, as it is shown by the arrows in the figure. It can be seen that region III is the only admissible area for a solution to satisfy the boundary condition $u(1)=\frac{\rho+\lambda+\mu(0)}{\lambda} p(1)$, which is on $L_{p}$. Indeed the only positive solutions must stay 
in that region and their path develops in the dotted area. Therefore, both $p(z)$ and $u(z)$ are decreasing functions: it follows that $p^{\prime}(z)<0$ and $u^{\prime}(z)<0$ everywhere. Moreover, in the same region, we have $p(z) \leq \frac{\lambda}{\rho+\lambda+\mu} u(z)<u(z)$ for all $z$.

\section{B.5. Proof of Proposition 2}

The results in Proposition 2 can be stated by investigating the upper-right plot in Fig. 1, where some qualitative features of the solution are represented by exploiting the paths describing two specific different geometric loci in $\left(\mathbb{R}^{+}\right)^{2}$. We define the following sets

$$
\begin{gathered}
L_{p}=\left\{(U, P) \in\left(\mathbb{R}^{+}\right)^{2} \mid \exists z \in[0,1] \text { so that } U=u(z)\right. \text { solves equations (13)-(14) } \\
\text { at the state } \left.z \text { and } P=\frac{\lambda}{\rho+\lambda+\mu(1-z)} U\right\}
\end{gathered}
$$

and

$$
\begin{gathered}
L_{u}=\left\{(U, P) \in\left(\mathbb{R}^{+}\right)^{2} \mid \exists z \in[0,1] \text { so that } U=u(z)\right. \text { solves equations (13)-(14) } \\
\text { at the state } \left.z \text { and } P=\frac{\rho+\lambda+\mu(z)}{\lambda} U\right\} .
\end{gathered}
$$

They can be considered as a generalization of the geometric loci already defined by (18) in Appendix B.4, where the monetary policy is assumed to be state independent. As a difference with respect to (18), here we consider an explicit dependence on the state $z$ for both $U$ and $P$ in the plane $\left(\mathbb{R}^{+}\right)^{2}:$ the sets are described by the points $\left(u(z), \Gamma_{p}(z):=\frac{\lambda}{\rho+\lambda+\mu(1-z)} u(z)\right) \in$ $L_{p}$ and $\left(u(z), \Gamma_{u}(z):=\frac{\lambda}{\rho+\lambda+\mu(z)} u(z)\right) \in L_{u}$, as $z$ varies in the domain [0,1]. Again the terms $p^{\prime}(z) h^{p}(z)$ and $u^{\prime}(z) h^{u}(z)$ in equations (13)-(14) are zero on $L_{p}$ and $L_{u}$, respectively. Then, according to the sign of terms $h^{p}(z)$ and $h^{u}(z)$, both $L_{p}$ and $L_{u}$ determine different regions in the plane $(U, P)$ where the functions $u(z), p(z)$ change behavior according to the sign of their derivative.

The following features are crucial to draw the loci $L_{p}, L_{u}$, and to characterize the solution of the differential problem assuming $A_{1}$ and $A_{2}$ :

$\left(P_{1}\right)$ The loci may intersect in an even number of points, since condition $\Gamma_{p}(z)=\Gamma_{u}(z)$ is equivalent to have $\rho^{2}+2 \rho \lambda+(\rho+\lambda)(\mu(z)+\mu(1-z))+\mu(z) \mu(1-z)=0$, which is a symmetric formula with respect to $z=1 / 2$.

$\left(P_{2}\right)$ Condition $\frac{\lambda}{\rho+\lambda+\mu(0)}<1 \leq \frac{\rho+\lambda+\mu(1)}{\lambda}$ in assumption $A_{1}$ can be extended in order to have the inequality $\frac{\lambda}{\rho+\lambda+\mu(1-z)} \leq \frac{\rho+\lambda+\mu(z)}{\lambda}$, satisfied in a suitable neighborhood of $z=1$. It follows that, in the same neighborhood, the locus $L_{p}$ is under $L_{u}$.

$\left(P_{3}\right)$ The boundary condition $u(1)=\frac{\rho+\lambda+\mu(0)}{\lambda} p(1)$ lies on $L_{p}$ (denoted by a bold dot in the figures). It has to be reached by the solution in correspondence with $z$ converging to 1 .

$\left(P_{4}\right) L_{p}$ and $L_{u}$ determine different regions in the phase plane $(U, P)$ where both functions $u(z)$, $p(z)$ change behavior according to their derivative sign. Since $L_{p}$ lies under $L_{u}$, as shown in the upper-right panel of Fig. 1, the local behavior of $u(z)$ and $p(z)$ in a neighborhood of $z=1$ can be determined as follows. Let the arrows describe the increasing/decreasing patterns for $u(z)$ and $p(z)$, accounting for the signs of $h^{u}(z)<0$ and $h^{p}(z)>0$ established by Lemma 2 . It is evident that $u(z)$ is decreasing when the money holdings of the productive 
agent is near $z=1$, since the solution must reach the boundary condition (the bold dot on $L_{p}$ ) according to $P_{3}$.

$\left(P_{5}\right)$ The increasing/decreasing shape for $L_{p}$ can be established by noticing that, for any $z \in[0,1], \Gamma_{p}^{\prime}(z)=\frac{\lambda}{\rho+\lambda+\mu(1-z)}\left(\frac{u(z) \mu^{\prime}(1-z)}{u^{\prime}(z)(\rho+\lambda+\mu(1-z))}+1\right) u^{\prime}(z)$. We remark that $\mu(1-z) \geq 0$ and $\mu^{\prime}(1-z)<0$ near $z=1$. The first condition is due to the continuity for $\mu(x)$ and $\mu(0) \geq 0$; the second relationship arises from assumption $A_{2}$. In this respect, since $u(z)$ is decreasing when $z$ moves near $1\left(\right.$ see $\left.P_{4}\right)$, then $u^{\prime}(z)<0$. It follows that $\Gamma_{p}^{\prime}(z)<0$ and the locus $L_{p}$ decreases in a neighborhood of $z=1$.

The previous features can be exploited in order to draw the picture in Fig. 1, whose inspection lets the proof of Proposition 2 be completed. It is evident that the only solutions that are positive must reach that boundary condition in the dotted region, that is under $L_{p}$ and $L_{u}$ (the dashed curve represents a possible path for the solution). Hence there exists a threshold $\bar{z} \in[0,1]$ such that for all $z \in I_{z}=[\bar{z}, 1]$ the solution develops in the region where both $p(z)$ and $u(z)$ are decreasing functions, that is equivalent to have $p^{\prime}(z)<0$ and $u^{\prime}(z)<0$. In the same region we have $p(z)<u(z)$. Indeed, since $\mu(0) \geq 0$ and $u(1)=\frac{\rho+\lambda+\mu(0)}{\lambda} p(1)$, the point $(u(1), p(1)) \in$ $L_{p}$ lies under the 45 degree line and $p(1)<u(1)$. Since the function $\mu$ is continuous then the inequality can be extended in $I_{z}$. This completes the proof.

\section{B.6. Proof of Proposition 3}

We prove that the complete markets allocation cannot be sustained when $\frac{\rho}{\lambda}>\bar{\rho}$ (i.e. when the expected duration of the cycle is sufficiently long). To this end it suffices to show that consumption is not equal to 1 at all values of the state $x \in[0,1]$. In particular let us consider $x=0$. The consumption function and the continuity of the consumption function give that $c^{u}(0)=\mu(0) p(1) / 2$. Recall that $\mu(0)$ is finite (since $\mu(x)$ is continuous over a compact set). The boundary condition in equation (12) can be rewritten as $p(1)=\frac{1}{\frac{\rho}{\lambda}+1+\frac{\mu(0)}{\lambda}} u(1)$. Since $u(1)$ is finite in any monetary equilibrium, it follows that $c^{u}(0)$ can be made arbitrarily small by increasing $\frac{\rho}{\lambda}$, i.e. that $\lim _{\rho / \lambda \rightarrow \infty} c^{u}(0)=0$. Therefore there exists a threshold $\bar{\rho}>0$ such that for all $\rho / \lambda \in(\bar{\rho}, \infty)$ we have that $c^{u}(0)<1$, or that the complete markets allocation cannot be implemented.

\section{Appendix C. Solving the ODEs and computing optimal policy}

Here we describe the algorithm used to solve for the optimal policy $\hat{\mu}(x)$. Given that we restrict our attention to piecewise continuous linear policy rules in $[0,1]$, the policy can be characterized by a vector with elements $a_{i}$ where $i$ indexes the number of nodes. More precisely, we assume that $\hat{\mu}(x)$ is continuous on $[0,1]$, it is linear on each subinterval $[(i-1) / I, i / I]$ for $i=1, \ldots, I$, and we set its nodal values as $\hat{\mu}(i / I)=a_{i}$ for each $i=0,1, \ldots, I$. For a given initial guess $v_{\text {old }}$ of the value function evaluated at $x=1 / 2$, the algorithm proceeds as follows:

1. Guess a set of values $a_{i}$ for all $i=0,1, \ldots, I$.

2. Solve the system given by equations (9), (10), (11), and (12). Details on the numerical implementation of the system are presented in Appendix C.1.

3. Compute the value function $v(1 / 2)$ in equation (16). Let $v_{\text {new }}=v(1 / 2)$. 
4. If $\left|v_{\text {new }}-v_{\text {old }}\right|<\epsilon$, then stop the algorithm and call $a_{i}$ the optimal policy $\hat{\mu}(x)$. If the inequality is not satisfied, let $v_{\text {old }}=v_{\text {new }}$ and return to step 1 .

To initialize the algorithm we let the initial $v_{\text {old }}$ be equal to the value under complete markets. Our initial guess of the policy rule is a constant rule (so that $a_{i}=a_{j}$ for all $i, j$ ). The optimization with respect to the draws for $a_{i}$ is performed following a simulated annealing algorithm. One advantage of the simulated annealing is that it is a global optimizer, which allows the optimization routine to deal with local maxima.

\section{C.1. Solving the system of ODEs}

Here we discuss the numerical implementation followed to solve the model. It is useful to rewrite the boundary value problem presented in equations (9), (10), (11), and (12) as we describe next. First the domain for $x \in[0,1]$, is halved and the following functions are defined

$$
y^{(1)}(z)=p(z), y^{(2)}(z)=u(z), y^{(3)}(z)=p(1-z), y^{(4)}(z)=u(1-z),
$$

for $z \in\left[\frac{1}{2}, 1\right]$. Thus, the evolution dynamics of the vector $y(z)=\left[y^{(1)}(z), y^{(2)}(z), y^{(3)}(z)\right.$, $\left.y^{(4)}(z)\right] \in \mathbb{R}^{4}$ is described by the following equation

$$
\dot{y}(z)=f(z, y(z)), \quad z \in\left[\frac{1}{2}, 1\right],
$$

where the forcing term entries are defined as

$$
\begin{aligned}
& f^{(1)}(z, y)=\frac{(\rho+\lambda+\mu(1-z)) y^{(1)}-\lambda y^{(2)}}{\mu(1-z)\left(\frac{1}{2}-z\right)+\frac{1}{y^{(4)}},} \\
& f^{(2)}(z, y)=\frac{(\rho+\lambda+\mu(z)) y^{(2)}-\lambda y^{(1)}}{\mu(z)\left(\frac{1}{2}-z\right)-\frac{1}{y^{(2)}}}, \\
& f^{(3)}(z, y)=-\frac{(\rho+\lambda+\mu(z)) y^{(3)}-\lambda y^{(4)}}{\mu(z)\left(z-\frac{1}{2}\right)+\frac{1}{y^{(2)}}}, \\
& f^{(4)}(z, y)=-\frac{(\rho+\lambda+\mu(1-z)) y^{(4)}-\lambda y^{(3)}}{\mu(1-z)\left(z-\frac{1}{2}\right)-\frac{1}{y^{(4)}}} .
\end{aligned}
$$

The boundary conditions are expressed as $F(y(1 / 2))=\underline{0}$ and $G(y(1))=\underline{0}$, where $\underline{0}=[0,0] \in \mathbb{R}^{2}$ and the functions $F, G$ map $\mathbb{R}^{4}$ onto $\mathbb{R}^{2}$ with entries

$$
F^{(1)}(w)=w^{(1)}-w^{(3)}, \quad F^{(2)}(w)=w^{(2)}-w^{(4)},
$$

and

$$
G^{(1)}(w)=w^{(4)}-\frac{2 \bar{c}}{\mu(0)}, \quad G^{(2)}(w)=w^{(2)}-\left(1+\frac{\rho}{\lambda}+\frac{\mu(0)}{\lambda}\right) w^{(1)} .
$$

Notice that expressing the boundary in this way imposes that $p(z)=p(1-z)$ and $u(z)=u(1-z)$ at $z=1 / 2$, i.e. that the $p$ and $u$ functions are continuous.

We adopt an $s$-stage explicit Runge-Kutta method $\left(a_{k j}, b_{k}, c_{k}\right)$ of order $s$ for integrating the system of differential equations. In the approximation, the interval $\left(\frac{1}{2}, 1\right)$ is discretized with a 
variable step-length $h_{n}$ and two sets of nodes: the primary nodes $z_{n+1}=z_{n}+h_{n}$, the intermediate nodes $z_{n_{k}}=z_{n}+c_{k} h_{n}\left(k=1, \ldots, s, c_{k} \leq 1\right)$ for $n=0, \ldots, N$, with $z_{0}=1 / 2$ and $z_{N}=1$. In the sequel, $y_{n}$ and $y_{n_{k}}$ denote the approximations for $y\left(z_{n}\right)$ and $y\left(z_{n_{k}}\right)$, respectively. We start from a pair of values $(\varsigma, v) \in \mathbb{R}^{2}$ and set $y(1 / 2)=y_{0}:=[\varsigma, v, \varsigma, v] \in \mathbb{R}^{4}$. Then, the discrete dynamics can be described with the following recursive formula

$$
\begin{aligned}
y_{n_{k}} & =y_{n}+h_{n} \sum_{j=1}^{k-1} a_{k j} f\left(z_{n_{j}}, y_{n_{j}}\right), \quad k=1, \ldots, s, \\
y_{n+1} & =y_{n}+h_{n} \sum_{k=1}^{s} b_{k} f\left(z_{n_{k}}, y_{n_{k}}\right),
\end{aligned}
$$

First the intermediate values $y_{n_{k}}$ are evaluated as defined in the first line of the previous scheme (20); then, they are used to compute the primary approximation $y_{n+1}$ as shown in the second line.

Since $F\left(y_{0}\right)=\underline{0}$ holds by the previous definition of $y_{0}$, what remains to be checked is that the boundary condition $G\left(y_{N}\right)=\underline{0}$ is satisfied. The scheme in (20) gives an explicit algorithm where the value for $y_{N}$ can be computed as soon as $y_{0}$ is known. Thus, we have to search for the pair $(\varsigma, v) \in \mathbb{R}^{2}$ such that $G\left(y_{N}\right)=\underline{0}$. This amounts to solving a system of two nonlinear equations with respect to the unknowns $\varsigma, v$. This procedure can be interpreted as a "shooting approach".

In particular, our numerical results are obtained by applying the so-called "classical RungeKutta method", which is very accurate since it features fourth order accuracy with respect to the step-lengths $h_{n}$. To find the pair $(\varsigma, v) \in \mathbb{R}^{2}$ which solves $G\left(y_{N}\right)=\underline{0}$, we use the following recursive procedure in order to evaluate $y_{N}$ :

$$
\begin{aligned}
& y_{0}=[\varsigma, v, \varsigma, v], \\
& y_{n_{1}}=y_{n}, \quad y_{n_{2}}=y_{n}+\frac{h_{n}}{2} f\left(z_{n_{1}}, y_{n_{1}}\right), \\
& y_{n_{3}}=y_{n}+\frac{h_{n}}{2} f\left(z_{n_{2}}, y_{n_{2}}\right), \quad y_{n_{4}}=y_{n}+h_{n} f\left(z_{n_{3}}, y_{n_{3}}\right), \\
& y_{n+1}=y_{n}+\frac{h_{n}}{6}\left(f\left(z_{n_{1}}, y_{n_{1}}\right)+2 f\left(z_{n_{2}}, y_{n_{2}}\right)+2 f\left(z_{n_{3}}, y_{n_{3}}\right)+f\left(z_{n_{4}}, y_{n_{4}}\right)\right),
\end{aligned}
$$

where the inner stages are

$$
\begin{aligned}
& z_{n_{1}}=z_{n}, \quad z_{n_{2}}=z_{n}+\frac{h_{n}}{2}, \\
& z_{n_{3}}=z_{n}+\frac{h_{n}}{2}, \quad z_{n_{4}}=z_{n}+h_{n}=z_{n+1}, \\
& z_{0}=1 / 2, \quad z_{N}=1 .
\end{aligned}
$$

In order to implement the previous algorithm we exploit the fsolve built-in Matlab function for solving the two equations and finding the values $\varsigma, v$.

We conclude by describing some features of this numerical procedure. A convenient property of the approach is that it requires to solve only two nonlinear equations in only two real variables $v, \varsigma \cdot{ }^{20}$ It must be noticed that, for the problem under scrutiny, any numerical algorithm

20 Other approaches could be pursued, such as methods that rely on classical Gaussian collocation. They solve a larger set of nonlinear equations in many unknowns. 
Table 2

Optimal policy $\mu(x)$ : robustness to number of nodes.

\begin{tabular}{|c|c|c|c|c|c|}
\hline & $\alpha$ & $\max \hat{\mu}(x)$ & $\arg \max \hat{\mu}(x)$ & $\min \hat{\mu}(x)$ & $\arg \min \hat{\mu}(x)$ \\
\hline \multicolumn{6}{|c|}{ Baseline, $\rho / \lambda=1 / 2$} \\
\hline 3 nodes & $9.45 \%$ & 0.06 & 0 & -0.13 & 0.5 \\
\hline 5 nodes & $3.31 \%$ & 0.59 & 0 & -0.52 & 0.5 \\
\hline 9 nodes & $3.28 \%$ & 0.59 & 0 & -0.53 & 0.5 \\
\hline 17 nodes & $3.25 \%$ & 0.61 & 0 & -0.57 & 0.5 \\
\hline \multicolumn{6}{|c|}{ Short cycles, $\rho / \lambda=1 / 10$} \\
\hline 3 nodes & $0.78 \%$ & 0.50 & 0 & -0.34 & 0.5 \\
\hline 5 nodes & $0.41 \%$ & 1.06 & 0 & -0.75 & 0.5 \\
\hline 9 nodes & $0.40 \%$ & 1.08 & 0 & -0.67 & 0.5 \\
\hline 17 nodes & $0.39 \%$ & 1.08 & 0 & -0.68 & 0.5 \\
\hline \multicolumn{6}{|c|}{ Long cycles, $\rho / \lambda=1$} \\
\hline 3 nodes & $13.63 \%$ & 0.11 & 0 & -0.21 & 0.5 \\
\hline 5 nodes & $11.69 \%$ & 0.20 & 0 & -0.33 & 0.5 \\
\hline 9 nodes & $9.61 \%$ & 0.23 & 0 & -0.35 & 0.5 \\
\hline 17 nodes & $9.57 \%$ & 0.23 & 0 & -0.35 & 0.5 \\
\hline
\end{tabular}

that exploits the direct evaluation of the forcing term in equation (14), has the drawback of evaluating the fraction defining $u^{\prime}(x)$ where the denominator nullifies as $x \rightarrow 0$, which corresponds to $z \rightarrow 1$ in equation (19). Two cases may arise: in the first case $u^{\prime}(x)$ diverges as $x \rightarrow 0$, as it happens with the constant money supply studied by Scheinkman and Weiss (1986). In this case the direct evaluation of the forcing term causes a failure in the algorithm. In the second case $u^{\prime}(x)$ is finite as $x \rightarrow 0$, that happens when both the numerator and the denominator are infinitesimal of the same order. Because the algorithm must perform divisions by a number that is close to zero, the approximation may give rise to oscillations that cause numerical instability and affect the whole solution. In general, for the discretization of equation (14) the instability arises in a neighborhood of $x=0$. In our approach, the approximation of equation (19) happens for $z \in\left(\frac{1}{2}, 1\right)$ and the instability arises at a point close to the boundary $z=1$, so that its propagation is confined at the end of the integration process. To deal with this, we exploit an adapted spatial mesh with a non-uniform length $h_{n}$, with a finer grid inside the integration interval, and a coarser grid near the boundary where $z=1$. The finer grid in the interior of the interval is chosen so as to have high accuracy of the approximation, while the coarser grid close to $z=1$ is chosen so as to minimize the number of fraction evaluations and the instability that occur near the boundary. ${ }^{21}$

\section{Appendix D. Robustness to the number of nodes}

In this section we explore the robustness of the optimal policy to the number of nodes for three different cases. The first case is our baseline parameterization of the model, where $\rho / \lambda=1 / 2$. The second case assumes that $\rho / \lambda=1 / 10$, which maps to business cycles of shorter duration relative to the baseline parameterization. The third case uses $\rho / \lambda=1$, which maps to business cycles of longer duration relative to the baseline parameterization. The results are presented in Table 2. In the table we present, for each of these three cases, some relevant features of the optimal policy $\hat{\mu}(x)$ for $3,5,9$ and 17 nodes. For each value of $\rho / \lambda$ and number of nodes, we

21 We verified, through numerical exploration, that a non-uniform spatial mesh with $N=4020$ primary nodes for $x$ was enough to tackle any propagation of unstable oscillations. 


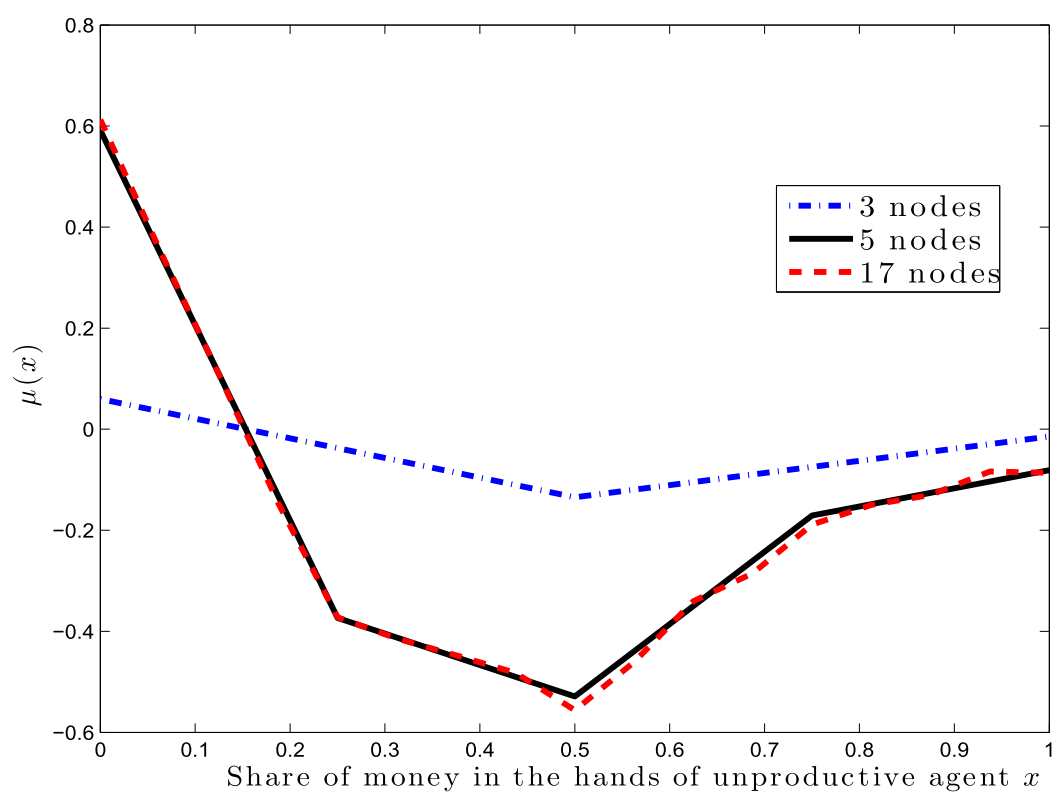

Fig. 7. Optimal policy: robustness to number of nodes (for $\rho / \lambda=0.5$ ).

present $\alpha$, the consumption equivalent cost of market incompleteness associated with a given policy (see equation (17)), the maximum and minimum values of the policy and the values of $x$ at which they occur. We present these particular statistics as we view them as particularly useful in gauging the robustness of the optimal policy to the number of nodes. Inspection of Table 2 reveals that the optimal policy $\hat{\mu}(x)$ stabilizes once the number of nodes reaches five. First, the welfare gains from increasing the number of nodes become very small. Second, there is no variation in terms of at which value of $x$ the policy is minimized and maximized. Third, there is also small variation of the maximum and minimum values attained by the optimal policy. Finally, in Fig. 7 we present, for the baseline parameterization, the policy rule $\mu(x)$ for 3, 5, and 17 nodes. To preserve visibility, we do not plot the policy with 9 nodes. Consistent with the results in the table, the figure shows that the policy stabilizes as we increase the number of nodes above 5 .

\section{Appendix E. Supplementary material}

Supplementary material related to this article can be found online at http://dx.doi.org/10.1016/ j.jet.2015.07.005.

\section{References}

Algan, Y., Challe, E., Ragot, X., 2011. Incomplete markets and the output-inflation tradeoff. Econ. Theory 46 (1), 55-84. Alvarez, F., Atkeson, A., Edmond, C., 2009. On the sluggish response of prices to money in an inventory-theoretic model of money demand. Q. J. Econ. 124 (3).

Andolfatto, D., 2010. Essential interest-bearing money. J. Econ. Theory 145 (4), 1495-1507.

Andolfatto, D., 2013. Incentive-feasible deflation. J. Monet. Econ. 60 (4), 383-390.

Ascher, U., Mattheij, R., Russell, R., 1988. Numerical Solution of Boundary Value Problems for Ordinary Differential Equations. Ser. Comput. Math. Prentice Hall. 
Atkeson, A., Lucas Jr., Robert E., 1992. On efficient distribution with private information. Rev. Econ. Stud. 59 (3), 427-453.

Berentsen, A., Camera, G., Waller, C., 2005. The distribution of money balances and the nonneutrality of money. Int. Econ. Rev. 46 (2), 465-487.

Bewley, T., 1980. The optimum quantity of money. In: Kareken, J., Wallace, N. (Eds.), Models of Monetary Economies. Federal Reserve Bank of Minneapolis, pp. 169-210.

Bhattacharya, J., Haslag, J.H., Martin, A., 2005. Heterogeneity, redistribution, and the Friedman rule. Int. Econ. Rev. 46 (2), 437-454.

Deviatov, A., Wallace, N., 2012. Optimal inflation in a model of inside money. Dept. of Economics, Penn State. Mimeo.

Green, E., Zhou, R., 2005. Money as a mechanism in a Bewley economy. Int. Econ. Rev. 46 (2), 351-371.

Guerrieri, V., Lorenzoni, G., 2009. Liquidity and trading dynamics. Econometrica 77 (6), 1751-1790.

Guerrieri, V., Lorenzoni, G., 2011. Credit crises, precautionary savings, and the liquidity trap. NBER working papers 17583. National Bureau of Economic Research, Inc.

Imrohoroglu, A., 1992. The welfare cost of inflation under imperfect insurance. J. Econ. Dyn. Control 16 (1), 79-91.

Kehoe, T.J., Levine, D.K., Woodford, M., 1992. The optimum quantity of money revisited. In: Dasgupta, P., Gale, D., Hart, O., Maskin, E. (Eds.), Economic Analysis of Markets and Games: Essays in Honor of Frank Hahn. MIT Press, pp. 501-526.

Kiyotaki, N., Moore, J., 1997. Credit cycles. J. Polit. Econ. 105 (2), 211-248.

Lagos, R., Wright, R., 2005. A unified framework for monetary theory and policy analysis. J. Polit. Econ. 113 (3), $463-484$.

Levine, D.K., 1991. Asset trading mechanisms and expansionary policy. J. Econ. Theory 54 (1), 148-164.

Lucas, R.J., 1990. Liquidity and interest rates. J. Econ. Theory 50 (2), 237-264.

Manuelli, R., Sargent, T.J., 2010. Alternative monetary policies in a turnpike economy. Macroecon. Dyn. 14 (05), $727-762$.

Molico, M., 2006. The distribution of money and prices in search equilibrium. Int. Econ. Rev. 47 (3), $701-722$.

Rocheteau, G., Weill, P.-O., Wong, T.-N., 2015. A tractable model of monetary exchange with ex-post heterogeneity. Working paper 21179. National Bureau of Economic Research.

Scheinkman, J.A., Weiss, L., 1986. Borrowing constraints and aggregate economic activity. Econometrica 54 (1), $23-45$. Shi, S., 1997. A divisible search model of fiat money. Econometrica 65 (1), 75-102.

Shi, S., 1999. Money, capital, and redistributive effects of monetary policies. J. Econ. Dyn. Control 23 (4), 565-590.

Stokey, N.L., Lucas, R.E., 1989. Recursive Methods in Economic Dynamics. Harvard University Press.

Townsend, R.M., 1980. Models of money with spatially separated agents. In: Kareken, J., Wallace, N. (Eds.), Models of Monetary Economies, pp. 265-304.

Wallace, N., 1981. A Modigliani-Miller theorem for open-market operations. Am. Econ. Rev. 71 (3), $267-274$.

Wallace, N., 1984. Some of the choices for monetary policy. Q. Rev. 8 (1).

Wallace, N., 2013. Optimal money-creation in 'pure-currency' economies: a conjecture. Q. J. Econ. 129, $259-274$.

Wallace, N., 2014. Optima in heterogeneous-agent monetary economies. Penn State University. Mimeo. 